\title{
Comparative analyses of Legionella species identifies genetic features of strains causing Legionnaires' disease
}

\author{
Laura Gomez-Valero ${ }^{1,2^{*}}$, Christophe Rusniok ${ }^{1,2}$, Monica Rolando ${ }^{1,2}$, Mario Neou ${ }^{1,2}$, Delphine Dervins-Ravault ${ }^{1,2}$, \\ Jasmin Demirtas ${ }^{1,2}$, Zoe Rouy ${ }^{9}$, Robert J Moore ${ }^{10}$, Honglei Chen ${ }^{10}$, Nicola K Petty ${ }^{3}$, Sophie Jarraud ${ }^{4,5}$, \\ Jerome Etienne ${ }^{4,5}$, Michael Steinert ${ }^{6}$, Klaus Heuner ${ }^{7}$, Simonetta Gribaldo ${ }^{8}$, Claudine Médigue ${ }^{9}$, Gernot Glöckner ${ }^{11}$, \\ Elizabeth L Hartland ${ }^{12}$ and Carmen Buchrieser ${ }^{1 *}$
}

\begin{abstract}
Background: The genus Legionella comprises over 60 species. However, L. pneumophila and L. longbeachae alone cause over $95 \%$ of Legionnaires' disease. To identify the genetic bases underlying the different capacities to cause disease we sequenced and compared the genomes of L. micdadei, L. hackeliae and L. fallonii (LLAP10), which are all rarely isolated from humans.

Results: We show that these Legionella species possess different virulence capacities in amoeba and macrophages, correlating with their occurrence in humans. Our comparative analysis of 11 Legionella genomes belonging to five species reveals highly heterogeneous genome content with over $60 \%$ representing species-specific genes; these comprise a complete prophage in L. micdadei, the first ever identified in a Legionella genome. Mobile elements are abundant in Legionella genomes; many encode type IV secretion systems for conjugative transfer, pointing to their importance for adaptation of the genus. The Dot/lcm secretion system is conserved, although the core set of substrates is small, as only 24 out of over 300 described Dot/lcm effector genes are present in all Legionella species. We also identified new eukaryotic motifs including thaumatin, synaptobrevin or clathrin/coatomer adaptine like domains.

Conclusions: Legionella genomes are highly dynamic due to a large mobilome mainly comprising type IV secretion systems, while a minority of core substrates is shared among the diverse species. Eukaryotic like proteins and motifs remain a hallmark of the genus Legionella. Key factors such as proteins involved in oxygen binding, iron storage, host membrane transport and certain Dot/lcm substrates are specific features of disease-related strains.
\end{abstract}

Keywords: Legionella, Genome, Legionnaires' disease, Mobilome, Eukaryotic like proteins

\section{Background}

Among the many pathogens provoking severe pneumonia, the Gram-negative bacteria Legionella pneumophila and Legionella longbeachae are responsible for Legionnaires' disease, a severe pneumonia that can be deadly if not treated promptly [1]. Although several of the more than 60 species described in the genus Legionella may cause disease, L. pneumophila is the major agent, responsible for nearly

\footnotetext{
* Correspondence: Igomez@pasteur.fr; cbuch@pasteur.fr

${ }^{1}$ Institut Pasteur, Biologie des Bactéries Intracellulaires, 28, rue du Dr. Roux, 75724 Paris, Cedex 15, France

Full list of author information is available at the end of the article
}

90\% of all cases worldwide. L. longbeachae comes second, causing around 2 to $7 \%$ of cases with the exception of Australia and New Zealand, where it is associated with 30\% of Legionnaires' disease cases [2]. Legionella micdadei, Legionella bozemanii, Legionella dumoffii, Legionella anisa, Legionella wadsworthii and Legionella feelei are rarely found in humans and the remaining Legionella species have never or only once been isolated from humans [2]. This highly significant difference in disease incidence among Legionella species may be due to different environmental distributions and/or to different virulence potential for humans. Few studies have analyzed the environmental 
distribution of Legionella, although one survey in France showed that L. pneumophila, which had a prevalence of $95.4 \%$ in clinical isolates, was found in only $28.2 \%$ of the environmental samples tested, whereas L. anisa was isolated in $13.8 \%$ of the environmental samples but found only once $(0.8 \%)$ in a clinical isolate [3]. Similarly, a more recent report from Denmark showed that only $4.5 \%$ of clinical cases were due to non-L. pneumophila strains and reported a strong discrepancy in the occurrence of different Legionella species in clinical and environmental isolates [4]. For example, L. anisa was highly abundant in the environment, but never found in clinical isolates. In contrast, L. bozemanni, L. longbeachae and L. micdadei were identified in clinical samples but never or rarely in environmental samples [4]. Furthermore, different Legionella species also seem to have a different host range and different capacities to infect human cells $[5,6]$. Taken together, independently of the environmental distribution, different Legionella species seem also to possess different abilities to infect eukaryotic cells and to cause disease in humans.

After publication of the L. pneumophila genome sequence in 2004 [7,8] and that of L. longbeachae in 2010 $[9,10]$ several additional L. pneumophila strains have been sequenced [11-14] as well as a few draft genome sequences of other species. However, apart from Legionella oakridgensis [15] none has been analyzed in detail. Thus the vast majority of comprehensively analyzed genome sequences are from the major human pathogens L. pneumophila (eight genomes) and L. longbeachae (two genomes). In order to deepen our insight into species never or rarely found in human disease, we completely sequenced and analyzed the genomes of three Legionella species, L. micdadei, Legionella hackeliae and Legionella fallonii (LLAP10), selected based on their different epidemiological characteristics compared with L. pneumophila and L. longbeachae. L. micdadei is found in less than $1 \%$ of community-acquired pneumonia [2], L. hackeliae has been isolated from humans only once [16], and L. fallonii has never been reported to cause disease. L. fallonii was originally designated LLAP10 for 'legionella-like amoebal pathogen 10' [17], a term coined by Rowbotham for bacteria that caused legionella-like infections in amoebae, but could not be grown on agar media.

Here we analyze and compare the L. micdadei, L. hackeliae and L. fallonii genomes and compare them with seven previously completely sequenced L. pneumophila (Paris, Philadelphia, Lens, Corby, Alcoy, Lorraine and HL06041035) $[7,8,11,14]$ and one L. longbeachae NSW150 genome sequence [9]. We confirm that the presence of 'eukaryotic-like proteins' (ELPs) is indeed a specific feature of the genus Legionella and extend the knowledge of these proteins further by identifying additional eukaryotic motifs. Analyses of the virulence of the different Legionella species in protozoan and human cells correlated with the genetic content and allowed us to identify specific features of human pathogenic Legionella and to define a core set of 24 type IV secretion system (T4SS) effectors present in the Legionella species examined to date.

\section{Results and discussion}

L. micdadei, L. hackeliae and L. fallonii show different virulence in amoeba or macrophages

Little to nothing is known about the environmental distribution and the virulence of different Legionella species for human cells. Similarly, it is not known why L. pneumophila and L. longbeachae are so predominant in human disease compared with other Legionella species. As a first step to understand these differences we analyzed the capacity of $L$. micdadei, L. hackeliae and $L$. fallonii to infect the protozoan species Acanthamoeba castellanii and the human monocytic cell line THP-1. As shown in Figure 1A, L. micdadei replicated in THP-1 cells, similar to L. pneumophila, while L. fallonii and L. hackeliae were unable to replicate in these cells, although they are phagocytosed efficiently as seen from the higher numbers entering the cells after one hour of infection (Figure 1A). In contrast, L. fallonii was able to replicate in $A$. castellanii (Figure 1B). However, neither L. hackeliae nor $L$. micdadei replicated in this amoeba. Thus, additional experiments are necessary to analyze whether A. castellani is their environmental host or not (Figure 1B). Similar results have been obtained using Dictyostelium discoideum as a host where L. micdadei can replicate in this model amoeba but L. hackeliae cannot [6]. In contrast, it was reported that $L$. micdadei is able to replicate in A. castellani $[6,18]$. Puzzled by these contradicting results we further analyzed the infection capacity of L. micdadei. Our infection assays had been carried out at $20^{\circ} \mathrm{C}$ whereas Hägele and colleagues [6] performed their infections at $30^{\circ} \mathrm{C}$. We thought that the different results might be due to the different temperatures used. We thus carried out infection assays at $30^{\circ} \mathrm{C}$ and also used amoeba plate testing [19] at $37^{\circ} \mathrm{C}$ and $30^{\circ} \mathrm{C}$ (Figure 1C). Indeed, L. micdadei was able to replicate in A. castellani at $37^{\circ} \mathrm{C}$ and also at $30^{\circ} \mathrm{C}$, although to a lesser extent compared with L. pneumophila (Additional file 1). This suggested that the replication capacity of $L$. micdadei in A. castellanii is temperature dependent.

Taken together the replication capacity of the different Legionella species in amoeba and human cells differed in a way similar to the epidemiological data for these species. This suggests that common as well as species-specific mechanisms might be involved in Legionella infection and replication in human cells.

\section{The Legionella genomes have similar genomic features but very different genome content}

At approximately $3.5 \mathrm{Mb}$, the genome sizes of L. hackeliae and L. micdadei are similar to that of L. pneumophila 

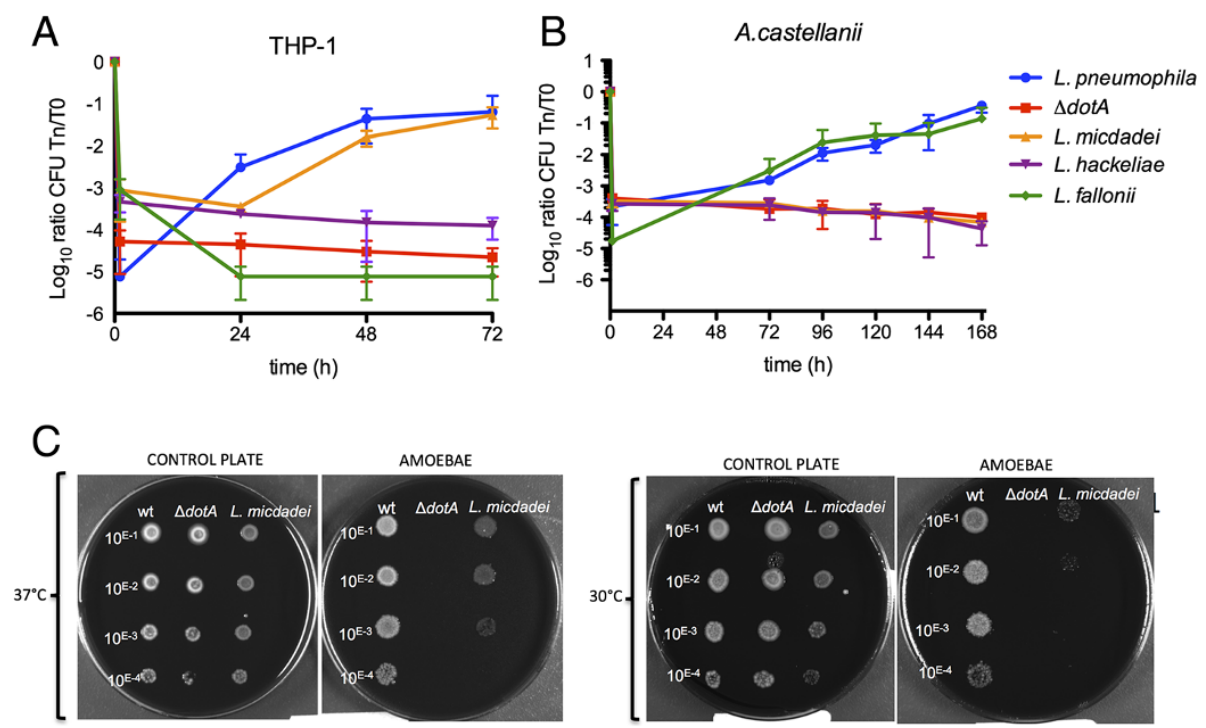

Figure 1 Intracellular replication of $L$. hackeliae, L. micdadei and L. fallonii (LLAP10). (A) THP-1 derived macrophages at $37^{\circ} \mathrm{C}$. (B) A. castellanii culture at $20^{\circ} \mathrm{C}$. (C) A. castellanii plate test at $37^{\circ} \mathrm{C}$ and $30^{\circ} \mathrm{C}$. L. pneumophila strain Paris wild type (wt) and $\Delta$ dotA were used as positive and negative controls, respectively. Intracellular replication for each strain was determined by recording the number of colony-forming units (CFU) through plating on BCYE agar. Blue, L. pneumophila strain Paris; red, $\Delta$ dotA; orange, L. micdadei; violet, L. hackeliae; green, L. fallonii (LLAP10). Results are expressed as Log 10 ratio CFU Tn/T0 and each point represents the mean \pm standard deviation of two or three independent experiments. The error bars represent the standard deviation, but some were too small to clearly appear in the figure.

whereas that of $L$. fallonii is similar to that of $L$. longbeachae at approximately $4 \mathrm{Mb}$ (Table 1 ). The GC content is highly homogenous (approximately 39\%) and the gene order is relatively well conserved. Apart from L. micdadei, each strain contained one or two plasmids between 14 and $238 \mathrm{~kb}$ in size (Table 1). When five different L. pneumophila genomes were compared the pan-genome comprised 2,957 genes, the core-genome of the species L. pneumophila contained 1,979 genes and the calculation of the rarefraction curves indicated that L. pneumophila has an open pan-genome [11]. This held true when we analyzed 11 Legionella genomes here (seven L. pneumophila strains and one strain each of $L$. longbeachae, $L$. micdadei, L. hackeliae and $L$. fallonii); the Legionella pan-genome increased considerably to 9,194 genes and the core genome was 1,388 genes (Figure 2A) or 1,415 genes when comparing one strain of each sequenced species (L. pneumophila Paris as representative) (Figure 2B). Thus, the core genome of Legionella represents only about $15 \%$ of the pan-genome, indicating that the Legionella accessory genome is large. The complete annotation of these three newly sequenced genomes is available in the LegionellaScope database [20] and at the Institut Pasteur, LegioList [21].

To establish a whole genome-based phylogeny of these Legionella species we used either 29 housekeeping genes or 816 orthologous genes shared among the 11 Legionella strains analyzed. Coxiella burnetii was used as outgroup. Phylogenetic reconstructions using either the nucleotide or the amino acid sequences gave the same tree topology for the different species. In contrast, the tree topology of the L. pneumophila strains was different depending on the data set or the phylogenetic method used, probably due to the high recombination rate of this species [12,22]. Our phylogenetic analyses showed that L. pneumophila, L. fallonii and L. longbeachae group together, with $L$. fallonii being phylogenetically the closest to L. pneumophila. L. micdadei and L. hackeliae formed a second cluster (Figure 3). Except for the place of L. fallonii, this is in agreement with previous phylogenies of the genus Legionella $[23,24]$. In previous work $L$. pneumophila was described as phylogenetically closer to L. longbeachae than to L. fallonii [25] or L. fallonii closer to L. longbeachae than to L. pneumophila [26]. However, these studies are based on 16S RNA sequences and bootstrap values associated with the corresponding nodes to evaluate its statistical support are not provided.

In conclusion, the general features of the Legionella genomes are very similar but each Legionella species has a distinctive genomic content with about $60 \%$ of genes being species-specific. Interestingly, human pathogenic and nonpathogenic species were mixed in the phylogeny, which indicates that virulent traits favoring human infection have been acquired independently during the evolution of the genus.

\section{Type II and IVB secretion systems are part of the core genome of Legionella}

Like in other bacterial genera, the core genome of Legionella contains the genes encoding fundamental 
Table 1 General features of the L. fallonii, L. micdadei and L. hackeliae genomes compared with L. pneumophila and L. longbeachae

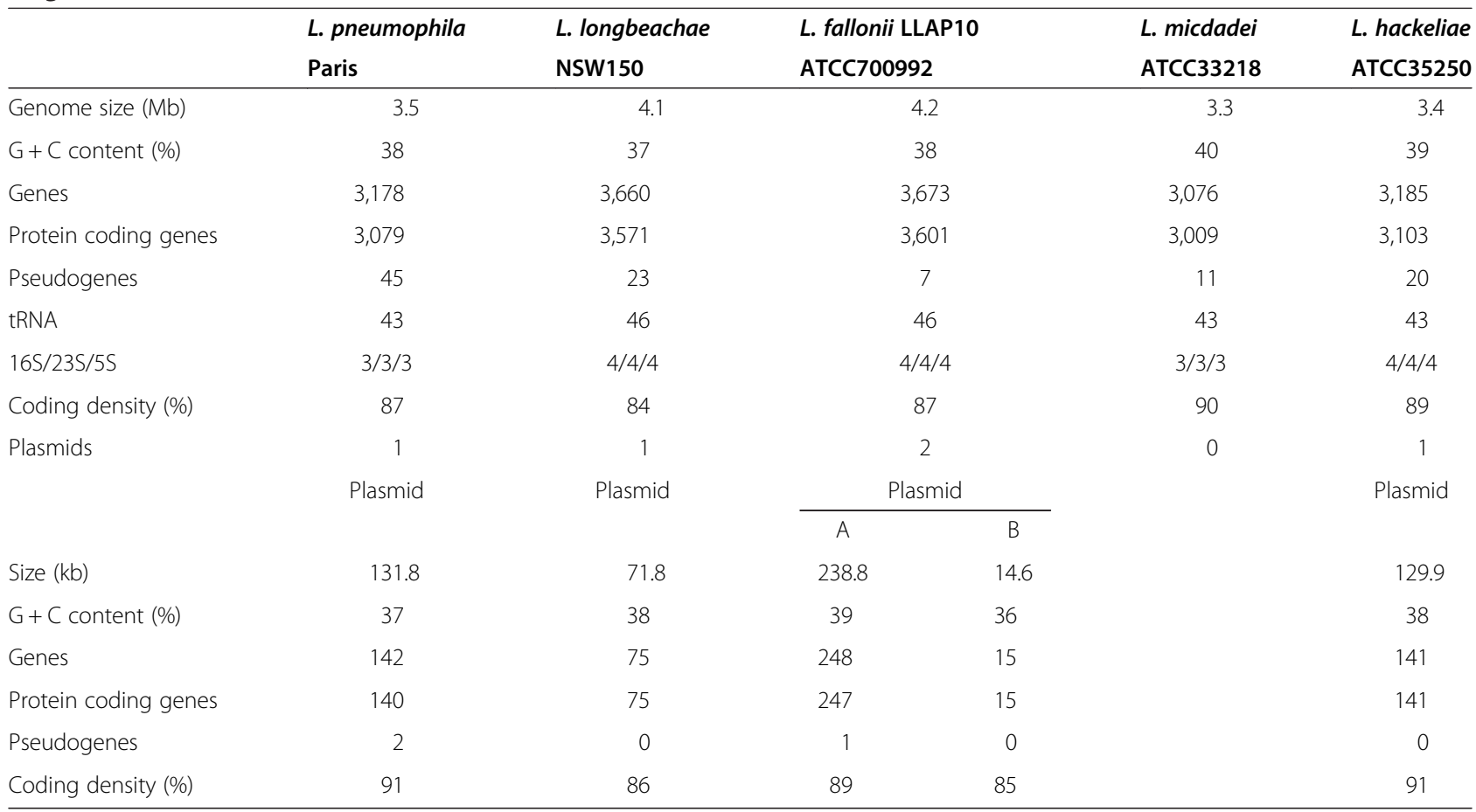

metabolic pathways and the ribosomal machinery. In addition, the Dot/Icm type IVB secretion system (T4BSS) as well as the Lsp type II secretion system (T2SS), both indispensable for intracellular replication, also belong to the core genome of this genus. The chromosomal organization of the Dot/Icm and the Lsp secretion system is also conserved, except for the genes $i c m D$ and $i c m C$, which are duplicated in L. fallonii. Interestingly, the degree of conservation of the different Dot/Icm proteins is very variable, ranging from $>90 \%$ for DotB to proteins without any homology such as IcmR. Surprisingly, DotA, an integral inner membrane protein
[27] indispensable for intracellular growth [28], is one of the least conserved proteins of the Dot/Icm T4SS (Additional file 2). Unexpectedly, the sequenced L. hackeliae strain (ATCC35250) had a stop codon in the gene coding for DotA, splitting it into 984 and 2,040 nucleotide fragments. Resequencing of the $\operatorname{dot} A$ gene confirmed the presence of the stop codon. As this strain was not able to replicate in $A$. castellanii, we thought that this might be due to the mutated $\operatorname{dot} A$ gene leading to a nonfunctional T4SS. To verify if this mutation was specific for the sequenced strain, we analyzed the $\operatorname{dot} A$ gene in a second L. hackeliae strain (ATCC35999). In this strain
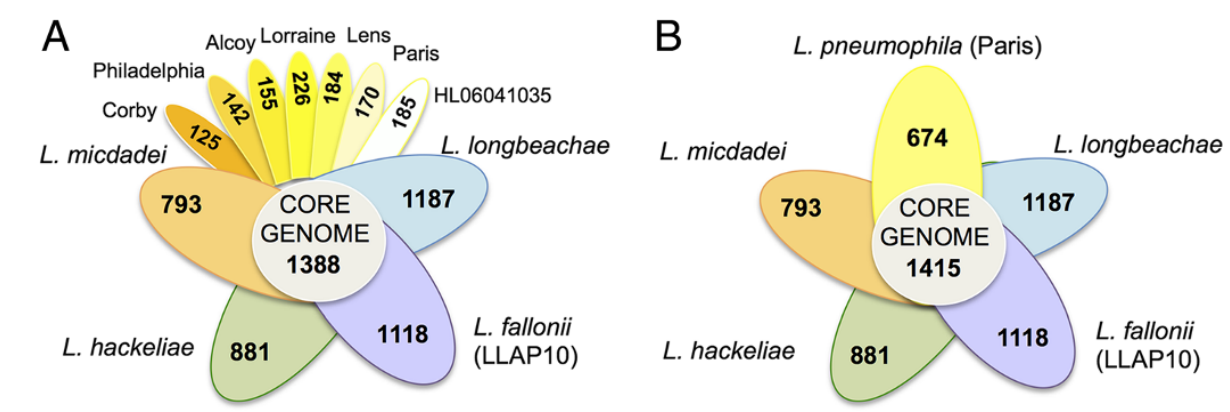

Figure 2 Shared and specific content of the different Legionella species/strains analyzed in this study. Each petal and color represents one genome. The number in the center of the diagram represents the orthologous genes shared by all the genomes. The number inside of each individual petal corresponds to the specific genes of each genome with non-orthologous genes in any of the other genomes. (A) Core genome of five Legionella species including seven L. pneumophila genomes. (B) Core genome when one representative of each Legionella species is taken into account. 


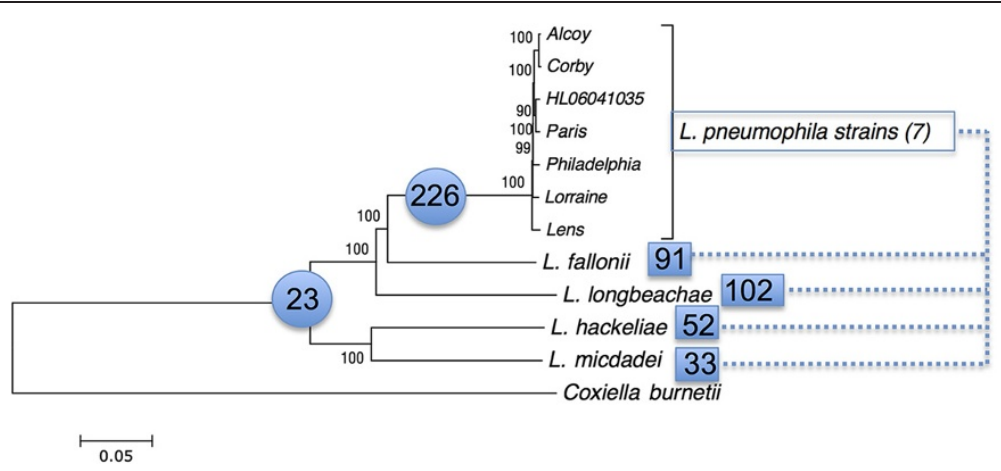

Figure 3 Phylogenetic tree of six Legionella species and seven L. pneumophila strains and their shared Dot/lcm substrates. Neighbor-joining tree based on the concatenation of 816 protein-coding genes from 11 Legionella genomes. C. burnetii was used as out-group. The tree was constructed using MEGA and JTT as model of evolution. The values above nodes indicate the bootstrap values. The values in blue circles represent the number of Dot/lcm substrates shared by the species in the corresponding cluster, suggesting that they were present in the common ancestor. The values inside blues squares are the number of Dot/lcm substrates shared between L. pneumophila strains and the remaining species (for example, the species L. micdadei and L. pneumophila share 33 Dot/lcm substrates).

the $\operatorname{dot} A$ gene was intact. Thus, the $\operatorname{dot} A$ gene fragmentation in the sequenced strain probably occurred during storage. However, when testing the virulence of both $L$. hackeliae strains in A. castellanii using the amoeba plate test, neither were able to replicate at $30^{\circ} \mathrm{C}$ or at $37^{\circ} \mathrm{C}$ (data not shown). To analyze if the Dot/Icm secretion system was functional in the sequenced strains, we used the calmodulin-dependent adenylate cyclase (CyaA) gene fusion approach [29] and RalF from L. pneumophila [30] for L. hackeliae, L. micdadei and L. fallonii. However, several attempts to show secretion of RalF in one of these strains failed, as RalF was never expressed in them despite testing under several different conditions. Thus, further experiments are necessary to adapt this assay to the here newly sequenced Legionella species.

Another particularity of the Dot/Icm system is the $i \mathrm{cmR}$ gene. Indeed, similar to what was reported for $L$. hackeliae and $L$. micdadei where $i c m R$ was replaced by a nonhomologous gene with functional equivalence [31,32], a gene encoding a protein with no similarity to any previously described protein is present in the position of $i \mathrm{~cm} R$ in L. fallonii, possibly serving as a functional equivalent of icmR of $L$. pneumophila. Other variable genes include $i \mathrm{~cm} X$ and $i \mathrm{~cm}$ G. IcmG has been described as a component that interacts with the effector proteins [33], which may explain the high variability in different species. In contrast, the components $\operatorname{dot} B, i \mathrm{~cm} S, i \mathrm{~cm} W$ and $i \mathrm{~cm} P$ are highly conserved. Indeed, these four genes can functionally replace their homologues in C. burnetii [34].

The L. micdadei, L. hackeliae and L. fallonii genomes encode surprising functions

L. fallonii is able to synthesize cellulose

Enzymes degrading cellulose have been described in L. longbeachae and were also found in L. fallonii. However, in addition the $L$. fallonii genome encodes a complete machinery for the synthesis of cellulose (Figure 4A). Although the bacterial need for cellulose may be surprising, cellulose has been reported as a common component of biofilms of several bacterial species such as Salmonella enterica or Escherichia coli [35]. The bacterial genes for cellulose synthesis are called bcsABZC. In S. enterica and E. coli a second operon necessary for cellulose biosynthesis named bcsEFG is present $[35,36]$. Both clusters (from lfa3354 to lfa3363 and lfa2987 to lfa2988) are present in L. fallonii, although with some differences in organization (Figure 4A). To analyze whether $L$. fallonii is able to synthesize cellulose, we used agar plates containing calcofluor, which binds cellulose and leads to fluorescence under UV radiation. Indeed, L. fallonii showed strong fluorescence under long-wave UV light, in contrast to L. pneumophila (Figure 4B), demonstrating cellulose biosynthesis in the genus Legionella for the first time. A blast search identified genes homologous to the L. fallonii cellulose operon (except $b c s E$ and $b c s F$ ) also in the draft genome sequences of $L$. anisa and $L$. dumoffii (Figure 4A). This suggests that several Legionella species have the capacity to synthesize cellulose.

\section{L. fallonii possesses genes encoding hopanoid biosynthesis and antibiotic resistance}

L. fallonii encodes genes for hopanoid biosynthesis currently not found in any other Legionella species. About $10 \%$ of all sequenced bacteria contain genes for hopanoid synthesis, in particular cyanobacteria, acetobacter, streptomycetes, methylotrophs and purple non-sulfur bacteria. Hopanoids have been proposed to enhance membrane stability and to decrease membrane permeability [37], similar to sterols in eukaryotic cell membranes [38]. In Burkholderia cenocepacia these genes are involved in sensitivity to low $\mathrm{pH}$, detergent and 


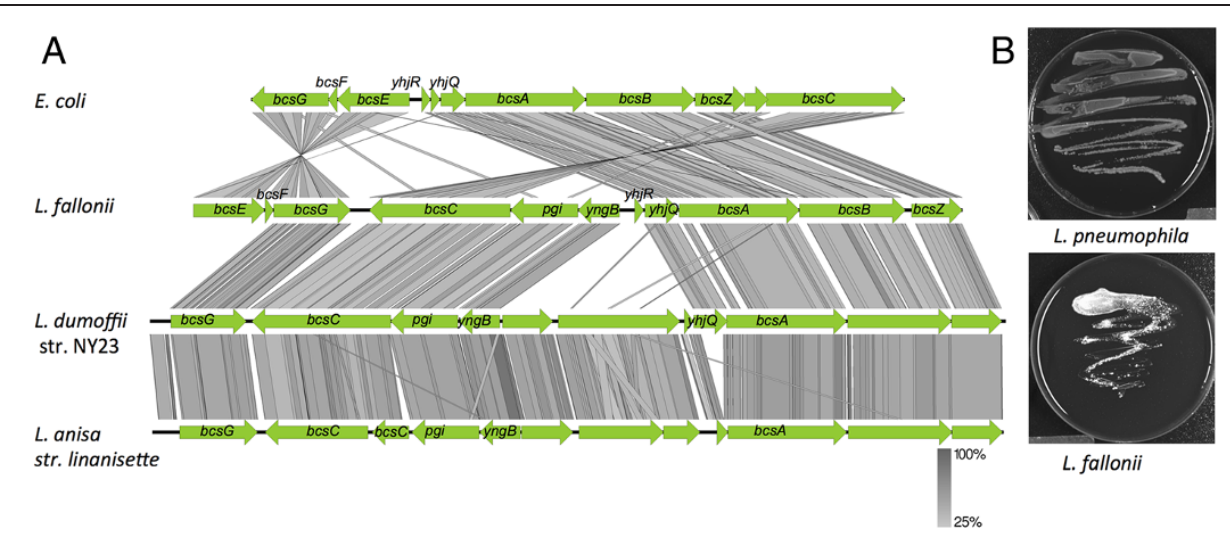

Figure 4 L. fallonii synthesizes cellulose. (A) Genomic organization and Blastx comparison of the regions encoding the cellulose synthesis machinery in E. coli, L. fallonii, L. dumofii and L. anisa. The gray color code represents the Blast matches; the darker the gray the better the blast match. (B) Growth of L. fallonii on calcofluor agar plates that shows cellulose synthesis as visualized under long-wave UV light. L. fallonii is fluorescent due to the binding of calcofluor to cellulose. In contrast L. pneumophila that was used as negative control is not.

antibiotics, and are related to motility [39]. In Streptomyces coelicolor, this cluster has been well studied. Although not all genes of the S. coelicolor cluster are conserved in L. fallonii (Additional file 3), to date all bacteria carrying the gene for squelene-hopene-cyclase produce hopanoids [39]. As L. fallonii also carries this gene, we expect this species is able to synthesize hopanoids, although their function in this species remains unknown.

Another peculiarity of L. fallonii is that it contains several antibiotic resistance genes not previously described in Legionella, including one encoding a chloramphenicol acetyltransferase (lfaO269) that is predicted to catalyze the acetyl-CoA-dependent acetylation of chloramphenicol. Furthermore, we identified a gene likely involved in erythromycin resistance, ereA (lfa1884) that is present also in $L$. drancourtii and $L$. dumoffii. This gene is located in gene clusters related to DNA mobility, such as integrases or prophage-related genes, and are rich in ELPs and repeats. These features indicate that these regions are putative genomic islands (Additional file 4).

\section{L. hackeliae and L. fallonii encode chitin deacetylase activity}

L. hackeliae and L. fallonii each contain a different gene coding for a chitin deacetylase (lha3256/lfa0697), an enzyme involved in deacetylation of chitin. An in vitro test described by Vadake [40] suggests that L. fallonii does have chitin deacetylase activity whereas it was not possible to demonstrate this clearly for $L$. hackeliae (Additional file 5). Chitin, a homopolymer of N-acetylglucosamine, is one of the most abundant polymers in the Earth's biomass, especially in marine environments. Interestingly it is also a component of the cyst wall of Entamoeba invadens, and enzymes responsible for chitin synthesis have been found in Entamoeba genomes [41].
The presence of chitin or chitin synthases has not been described in other protozoan genomes, but very few genomes of this group have been sequenced yet. Thus, chitin may be a common component of protozoa that are able to encyst. Although the other Legionella genomes analyzed here do not encode chitin deacetylase activity, all Legionella genomes encode chitinases. Chitinases are chitin-degrading enzymes leading to low molecular weight chito-oligomers whereas chitin decetylase degrades chitin to chitosan. Both products are of interest for industry and there is growing interest in organisms that produce chitosan. Legionella may be a new possible source of chitosan production.

\section{L. micdadei contains the first putative complete prophage identified in a Legionella genome}

The analysis of the unique genes from $L$. micdadei identified a specific region encoding 73 proteins, at least 16 of which are phage-associated proteins that represent a putative complete prophage (Additional file 6). This region contains genes encoding the phage capsid tail and replication proteins. Complete prophages have never been described in Legionella despite the frequent presence of phage-related proteins scattered in their genomes. Most attempts to isolate prophages that exclusively infect Legionella have also failed, until recently when two groups isolated Legionella bacteriophages [42,43] from environmental water samples and organs of guinea pigs. Thus, Legionella do have phages, but they seem to be rare.

\section{L. fallonii and L. micdadei contain additional flagella operons}

The comparison of the L. pneumophila and L. longbeachae genomes revealed that $L$. longbeachae does not contain genes allowing flagella biosynthesis [9]. As recognition of 
flagellin by Naip5 initiates host immune responses that control L. pneumophila infection in certain eukaryotic cells $[44,45]$, the presence or absence of flagella is important for intracellular replication of Legionella. L. hackeliae, L. fallonii and L. micdadei also contain three flagella operons homologous to those described in L. pneumophila (Figure S5A-C in Additional file 7). Interestingly L. fallonii and $L$. micdadei encode a fourth region not previously described in any sequenced Legionella species that might also code for flagella (Figure 5).

\section{A highly dynamic mobilome characterizes the Legionella genomes}

Genomic elements like plasmids, genomic islands or transposons constitute the mobilome of a genome. All Legionella species analyzed contain many of these mobile elements. For example, $L$. hackeliae possesses a plasmid of $129.88 \mathrm{~kb}$ whereas L. fallonii (LLAP10) contains two plasmids of $238.76 \mathrm{~kb}$ and $14.57 \mathrm{~kb}$, respectively (Table 1). Furthermore, the plasmid present in L. hackeliae is identical to the L. pneumophila strain Paris plasmid (100\% nucleotide identity over the entire length except for two transposases in the strain Paris plasmid; Additional file 8). This suggests that this plasmid has recently moved horizontally between both species, which is a new example of the high rate of gene transfer among Legionella genomes [46,47].

In addition to the plasmids identified and their evident exchange among strains and species, a hallmark of the
Legionella mobilome is the presence of many different type IVA secretion system-encoding regions in the plasmids as well as in genomic island-like regions on the chromosome. Interestingly, these regions often encode tra-like genes with considerable homology among the different strains. However, each new strain analyzed contained new regions, underlining the high diversity of these systems in the Legionella genomes. Predominant are F-type and P-type IVA systems that code for conjugative pili that allow mating. F-type IVA secretion systems are present on all L. pneumophila plasmids, the L. hackeliae plasmid, the $238 \mathrm{~kb}$ L. fallonii plasmid (two systems) and on the chromosomes of L. pneumophila strain Philadelphia, L. longbeachae and L. fallonii (Additional file 9). Each encodes a homologue of the global regulator CsrA, named $\mathrm{LvrC}$, which when present in the chromosome also encodes the $\operatorname{lv} R A B$ gene cluster. This was recently described as being involved in the regulation of excision of the ICE Trb1 of L. pneumophila strain Corby [48]. Thus, conjugative exchange of DNA has an important role in Legionella and is one key factor enabling Legionella to rapidly adapt to changing conditions.

The mobility and horizontal transfer of these different regions are further shown when studying the distribution of these systems. For example, the $l v h$ cluster, a type IVA system involved in virulence under conditions mimicking the spread of Legionnaires' disease from environmental niches [49], is also present in L. micdadei, in one of the two completely sequenced $L$. longbeachae strains
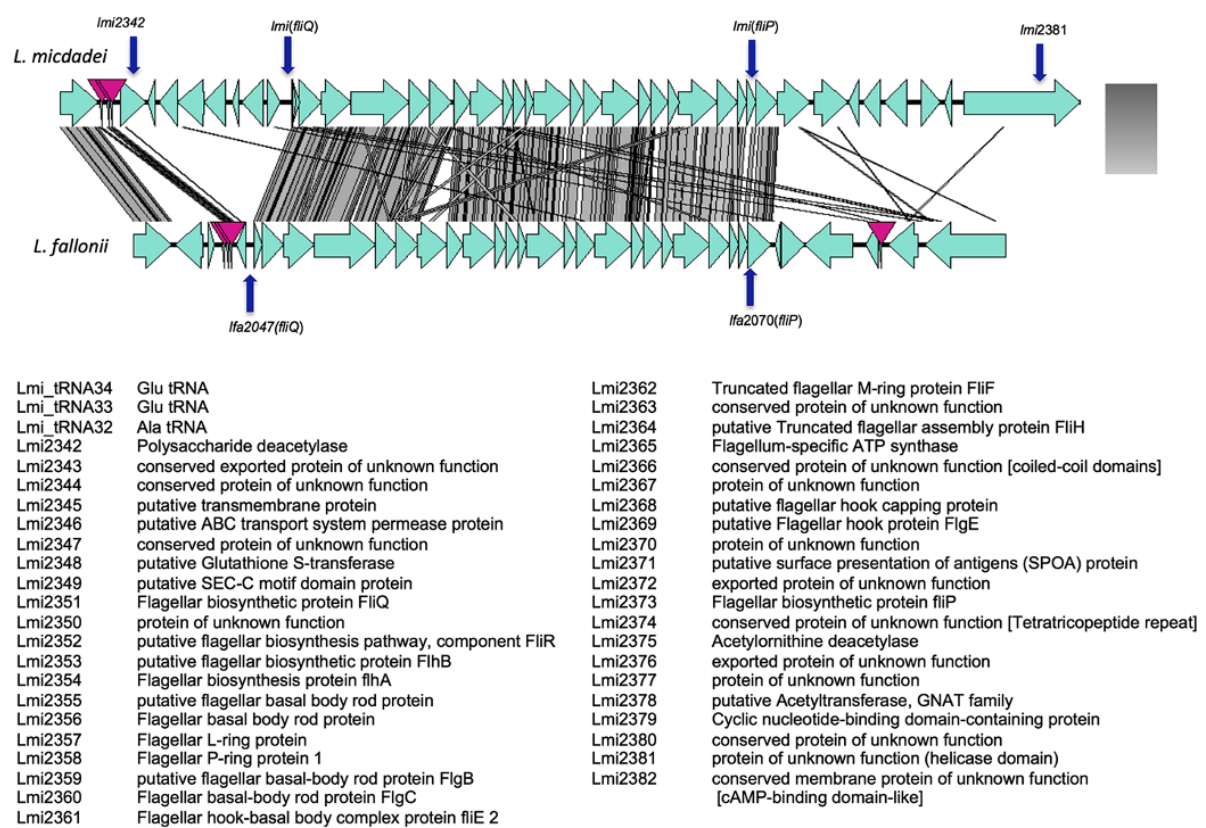

Figure 5 The $L$. micdadei and $L$. fallonii genomes contain specific flagellar-encoding regions. Genomic organization and Blastx comparison of the specific flagellar gene clusters in L. micdadei and L. fallonii. The gray color code represents the Blast matches; the darker the gray the better the blast match. Pink arrows point to tRNA genes. Protein names and their predicted function in L. micdadei are indicated below. 
and in five of the completely sequenced L. pneumophila strains (Table 2). In addition, the so-called GI-T4SS recently described in strain L. pneumophila 130b [13], and first recognized in Haemophilus influenzae as a T4SS involved in propagation of genomic islands [50], is believed to play an important role in the evolution and adaptation of Legionella [51]. GI-T4SS clusters were found to be conserved in L. pneumophila, with two clusters each in strains Corby, Paris, 130b and HL06041035, and one in each of Alcoy, Philadelphia, Lens and Lorraine [51], as well as in strains of L. longbeachae, L. hackeliae, L. micdadei and L. fallonii (Table 2). Thus, a heterogeneous distribution among species and strains testifies to the continuous exchange of these elements among Legionella, contributing to the plasticity and dynamic nature of their genomes.

\section{L. micdadei strains from different geographical regions are highly similar except for their mobilome}

To investigate the genomic diversity of the species L. micdadei, we determined the draft genome sequence of a clinical isolate obtained from the Microbiological Diagnostic Unit Public Health Laboratory (MDU), Australia and compared it with the completely sequenced strain L. micdadei ATCC 33218. The genome

Table 2 Distribution of type IV secretion systems in the analyzed Legionella genomes

\begin{tabular}{|c|c|c|c|c|c|c|}
\hline \multirow{2}{*}{\multicolumn{2}{|c|}{$\begin{array}{l}\text { Type IV secretion systems } \\
\text { Chromosome }\end{array}$}} & \multirow{2}{*}{$\begin{array}{l}\text { Type IVB } \\
\text { Dot/Icm }\end{array}$} & \multicolumn{3}{|c|}{ Type IVA } & \multirow[t]{2}{*}{ Gl-type } \\
\hline & & & P-type & F-type & Lvh & \\
\hline \multirow[t]{7}{*}{ L. pneumophila } & Paris & 1 & - & - & 1 & 2 \\
\hline & Lens & 1 & - & - & 1 & 1 \\
\hline & Philadelphia & 1 & - & 1 & 1 & 1 \\
\hline & Corby & 1 & 2 & - & - & 2 \\
\hline & Lorraine & 1 & 1 & - & - & 1 \\
\hline & HL06041035 & 1 & - & - & 1 & 2 \\
\hline & $130 b$ & 1 & 1 & & 2 & 2 \\
\hline L. longbeachae & NSW-150 & 1 & 1 & 1 & - & - \\
\hline L. longbeachae & D-4968 & 1 & - & 1 & 1 & 1 \\
\hline L. hackeliae & ATCC 35250 & 1 & - & - & - & 1 \\
\hline L. micdadei & ATCC33218 & 1 & - & - & 1 & 1 \\
\hline L. micdadei & $02 / 42$ & 1 & 1 & - & 1 & - \\
\hline $\begin{array}{l}\text { L. fallonii } \\
\text { (LLAP10) }\end{array}$ & ATCC700992 & 1 & - & 1 & - & 1 \\
\hline \multicolumn{7}{|l|}{ Plasmids } \\
\hline \multirow{3}{*}{$\begin{array}{l}\text { L. } \\
\text { pneumophila }\end{array}$} & Paris & - & - & 1 & - & - \\
\hline & Lens & - & - & 1 & - & - \\
\hline & Lorraine & - & - & 1 & - & - \\
\hline L. hackeliae & & - & - & 1 & - & - \\
\hline L. fallonii & & - & - & 2 & - & - \\
\hline
\end{tabular}

size and GC content of the two L. micdadei strains were highly similar (Figure 6). The main differences between the two L. micdadei strains were mobile genetic elements. Furthermore, the number of SNPs (1,985 SNPs) was very low, similar to serogroup 1 strains of $L$. longbeachae (1,611 SNPs) [9]. This is strikingly different to L. pneumophila where two different strains may contain more than 30000 SNPs. This suggests that L. micdadei and $L$. longbeachae evolved more recently compared to the L. pneumophila. Three large regions of the L. micdadei ATCC 33218 genome are absent from the Australian isolate (Figure 6). One is a genomic island encoding a GI-T4SS (36 kb), one is the predicted prophage we identified in this study, and another is a smaller cluster of approximately $9 \mathrm{~kb}$ that is flanked by three tRNA genes and that contains phage-related genes and a gene associated with abortive infection system (Figure 6). Similarly, in the Australian isolate a cluster absent from the completely sequenced $L$. micdadei strain corresponds to a P-type IVA secretion system. Interestingly, the Lvh region, encoding a T4ASS that is highly conserved among all strains and species analyzed to date, is divergent in the two L. micdadei strains with a high number of SNPs (Additional file 10). Thus, the main genetic differences between these two closely related $L$. micdadei strains are mobile genetic elements, further underlining the large extent of horizontal gene transfer that is present in the genus Legionella.

\section{The core set of Dot/lcm effectors is small with only 24 conserved substrates}

L. pneumophila encodes over 300 proteins that are translocated into the host cell by the Dot/Icm T4SS (Additional file 11). Their conservation is high among different L. pneumophila strains, as $77 \%$ of these substrates are present in all L. pneumophila strains sequenced to date. Interestingly, when the Dot/Icm substrates of L. pneumophila and L. longbeachae are compared, only 35\% (101) are present in both species [9]. Interestingly, the L. longbeachae and L. pneumophila genomes contain the highest number of common substrates, although L. fallonii is phylogenetically closer to L. pneumophila than to L. longbeachae (Figure 3). When investigating the presence of these substrates in five Legionella species by adding the L. hackeliae, L. micdadei and L. fallonii genomes, this revealed that their conservation is very low (Figure 3 ). With 33 conserved substrates, the lowest number is shared between L. micdadei and L. pneumophila. This result suggests that the shared substrates might relate to similar environmental niches or virulence properties (L. pneumophila and L. longbeachae) than to a closer phylogenetic relationship.

The Dot/Icm substrates conserved in all Legionella species are probably those indispensable for intracellular 


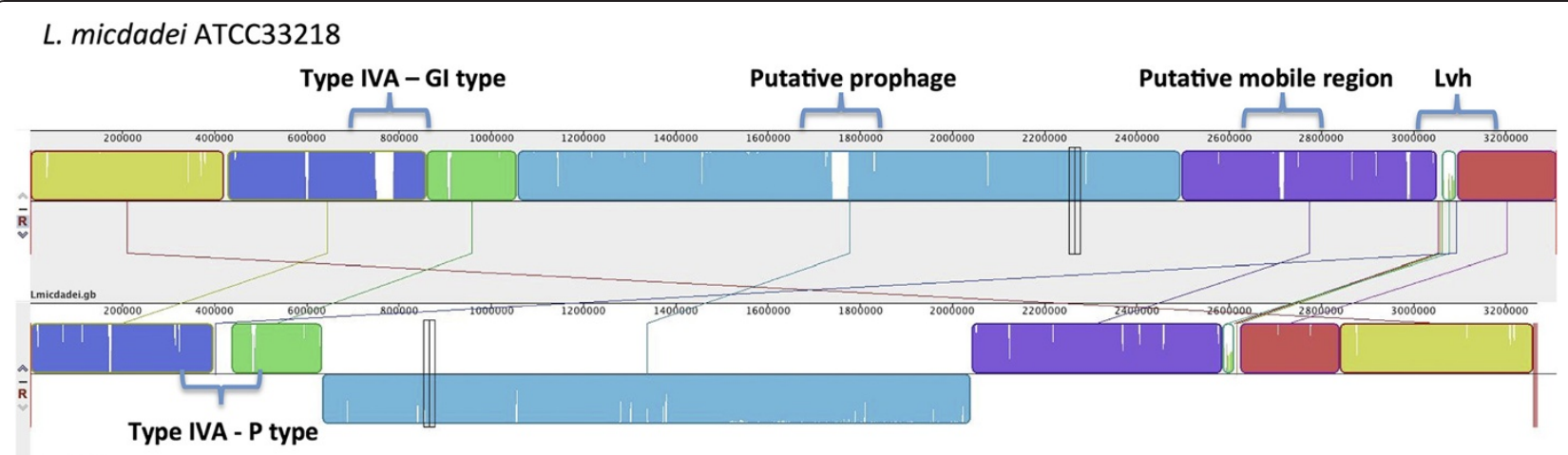

\section{L. micdadei clinical isolate Australia}

Figure 6 Genome comparison of two L. micdadei strains. The complete genome sequences of the two L. micdadei strains included in this study were aligned using the software Mauve. The two strains align perfectly with the exception of three mobile genetic elements specifically present in strain L. micdadei ATCC33218 and one specifically present in the Victorian isolate. The specific regions of each genome are indicated. The 'Lvh region' is indicated, as this region is, with a high number of SNPs, quite divergent between the two isolates.

replication and are important players in host-pathogen interactions. Most surprisingly, only 24 of the 300 described substrates of L. pneumophila are present in all five Legionella species and most of these are of yet unknown function (Table 3). However, a third of the conserved substrates contain eukaryotic motifs like ankyrin or Sel-1 domains or TPR repeats. Others were previously defined as ELPs, such as the sphingomyelinase-like phosphodiesterase. Among the substrates that have been investigated further are VipF, which causes growth defects in S. cerevisae, and several of the ankyrin repeat motif proteins. VipF inhibits lysosomal protein trafficking [52] and AnkH was shown to play a role in intracellular replication of $L$. pneumophila in macrophages and protozoa and in intrapulmonary proliferation in mice [53]. The function of MavBFNQ and RavC is not known, but they have been recovered in screens for vacuolar localization and have been shown to co-localize with SidC at the L. pneumophila vacuole [54].

SdhA, a L. pneumophila effector that is necessary for full virulence of this species, is a particular case. It is present in all Legionella analyzed but the similarity with L. longbeachae is small and thus below the cutoff established for our orthologous search (at least $65 \%$ of the length of the compared protein). However, given that homologues with a significant similarity are present in all species in synteny (except for L. hackeliae), and coiled-coil motifs are detected in all, SdhA was also defined as a core effector. Moreover, SdhA has been shown to be necessary for infection of mice and in Galleria mellonella [55,56]. Surprisingly, the effector SidJ is not part of the core set of Legionella substrates, although its deletion led to a strong replication defect in eukaryotic cells. However, SidJ is present in L. pneumophila and L. longbeachae, the major human pathogens.
Interestingly, the growth defect of strains lacking SdhA and SidJ seems more important in mice and human macrophages than in amoeba. Replication of the $s d h A$ mutant is severely impaired in mouse bone marrow-derived macrophages but less in the amoeba Dictyostelium discoideum [56]. Similarly, a $\Delta s i d J$ strain shows significant growth defects in both macrophages and amoebae, but replication in macrophages is affected from the start of the infection, whereas the growth defect in amoebae is evident only after $72 \mathrm{~h}$ of infection and was less pronounced [57]. These data may suggest that effectors important in human infection are not necessarily essential in the protozoan hosts and thus certain effectors might be important for human infection even though no growth defect in protozoan infection is detectable.

\section{Eukaryotic-like proteins are a specific feature of the genus Legionella}

One feature shared by many of the substrates of the Dot/ Icm secretion system is the presence of eukaryotic motifs (EMs). Indeed, of 55 proteins of L. pneumophila Philadelphia encoding EMs, 45 (82\%) are confirmed substrates of the Dot/Icm secretion system (Additional file 12). Thus, we searched for proteins containing EMs in all sequenced genomes. In the five Legionella species we identified 218 proteins with eukaryotic domains (Additional file 13). The genomes of $L$. longbeachae and $L$. fallonii contain nearly twice as many proteins with EMs as the other genomes, probably due to their larger genome size. The ankyrin motif is the most frequent one, followed by long coiledcoil domains. Some EMs that were described remain specific for $L$. longbeachae, such as the PPR repeats, PAM2 domain or the phosphatidylinositol-4-phosphate 5-kinase, indicating that they are probably related to its particular habitat in soil [9]. In contrast, proteins with tubulin- 
Table 3 Core of substrates of the Dot/lcm secretion system present in 11 Legionella genomes

\begin{tabular}{|c|c|c|c|c|c|c|}
\hline \multirow[t]{2}{*}{ Label } & \multirow[t]{2}{*}{ Product and associated motif } & \multirow[t]{2}{*}{ Gene name } & \multicolumn{4}{|c|}{ Amino acid identity ${ }^{a}$} \\
\hline & & & L. fallonii & L. long. & L. micdadeii & L. hack \\
\hline Ipg0021//pp0021 & Protein of unknown function [ribosome associated protein] & & 75 & 75 & 68 & 68 \\
\hline lpg0103/lpp0117 & N-terminal acetyltransferase, GNAT family & vipF & 62 & 61 & 50 & 47 \\
\hline Ipg0107//pp0121 b & Conserved protein of unknown function & $\operatorname{rave}$ & 84 & 80 & 72 & 47 \\
\hline Ipg0140/lpp0155 & Membrane protein of unknown function & $\operatorname{cet} L P 1$ & 53 & 45 & 39 & 36 \\
\hline lpg0376//pp0443 & Protein of unknown function & $s d h A$ & 60 & 48 & 38 & 38 \\
\hline Ipg0405//pp0471 & Protein of unknown function & & 56 & 56 & 50 & 40 \\
\hline lpg0483/lpp0547 & Protein of unknown function [ankyrin $]^{c}$ & legA12/ankC & 55 & 49 & 31 & 29 \\
\hline Ipg1356/lpp1310 & Putative Beta-lactamase [Sel1-domain $]^{c}[T P R \text { repeat }]^{c}$ & EnhC homologue & 80 & 71 & 67 & 69 \\
\hline lpg1578//pp4178 & Protein of unknown function & & 50 & 37 & 25 & 24 \\
\hline lpg1661//pp1632 & Heparan-alpha glucosaminide $\mathrm{N}$-acetyltransferase ${ }^{d}$ & & 60 & 57 & 47 & 48 \\
\hline Ipg1663//pp1634 & Protein of unknown function & cetLP3 & 54 & 44 & 40 & 58 \\
\hline lpg1752/lpp1716 & Protein of unknown function & mavB & 41 & 40 & 27 & 26 \\
\hline lpg1907//pp1882 & Membrane protein of unknown function [hydrolases] & & 54 & 52 & 44 & 49 \\
\hline lpg2300/lpp2248 & Protein of unknown function [ankyrin] ${ }^{c}$ & legA3/ankH/ankW & 81 & 76 & 71 & 75 \\
\hline lpg2322//pp2270 & Protein of unknown function [ankyrin] & legA5/ankK & 57 & 51 & 39 & 42 \\
\hline lpg2351//pp2300 & Protein of unknown function & mavF & 56 & 50 & 33 & 33 \\
\hline lpg2359//pp2308 & Protein of unknown function [putative GatB/Yqey domain] ${ }^{c}$ & & 82 & 79 & 73 & 72 \\
\hline lpg2588//pp2947 & Acid sphingomyelinase-like phosphodiesterase & legS1 & 64 & 61 & 54 & 33 \\
\hline lpg2591//pp2644 & Protein of unknown function & $\operatorname{ceg} 33$ & 46 & 47 & 34 & 26 \\
\hline lpg2815//pp2867 & Membrane protein of unknown function & mavN & 62 & 64 & 54 & 52 \\
\hline lpg2832//pp2889 & Protein of unknown function & & 72 & 68 & 60 & 62 \\
\hline lpg2888//pp2947 & Membrane protein of unknown function [coiled-coil] ${ }^{c}$ & & 59 & 50 & 34 & 40 \\
\hline lpg2936//pp3004 & Ribosomal RNA small subunit methyltransferase $\mathrm{E}$ & & 67 & 67 & 60 & 60 \\
\hline Ipg2975//pp3047 & Protein of unknown function & mave & 58 & 57 & 33 & 30 \\
\hline
\end{tabular}

${ }^{\mathrm{a}}$ Amino acid identity with respect to $L$. pneumophila strain Philadelphia. ${ }^{\mathrm{b}} \mathrm{ravC}$ has been erroneously named as Ipg0170 in previous publications. ${ }^{\mathrm{C}}$ The motif is present in all orthologues. ${ }^{\mathrm{d}}$ High prediction for enzymatic activity in all the corresponding orthologues (prediction based on PRIAM EC number). Bold numbers indicate synteny between $L$. pneumophila genes and the corresponding orthologous gene.

tyrosine ligase domains (LLo2200), probably involved in the posttranslational modification of tubulin [58], are absent only from L. pneumophila. With the aim to analyze whether additional eukaryotic motifs not yet identified are present in the Legionella genomes, we developed a strategy allowing for a comprehensive scan of all genomes. First we searched the Interpro database for all motifs, which occur in at least $85 \%$ of proteins from eukaryotic genomes and only $15 \%$ or less in proteins from prokaryotic genomes. Using this criterion, we obtained 8,329 motifs that were considered as eukaryotic (see Materials and methods). All predicted Legionella proteins were scanned for these motifs. This approach allowed us to identify 10 EMs not described before in Legionella, including thaumatin, RhoGTPase and DM9 domains (Table 4). Interestingly, thaumatin-like proteins accumulate in plants in response to infection by pathogens and possess antifungal activity $[59,60]$ and a Drosophila DM9-containing protein is strongly up-regulated after infection of Drosphila larvae by Pseudomonas species [61]. Many of these new EMs are only present in the newly sequenced genomes, such as synaptobrevin, an intrinsic membrane protein of small synaptic vesicles [62] or the clathrin/coatomer adaptinelike domain that is associated with transport between the endoplasmic reticulum and Golgi [63]. Given their function in eukaryotic organisms, these protein domains might indeed be important in host-pathogen interactions.

\section{Many eukaryotic proteins are indeed transferred horizontally from eukaryotes}

Not all proteins we defined as ELPs possess EMs, but certain ones are also considered eukaryotic-like as they show a high homology to eukaryotic proteins over their whole length. One of the best known examples of this type of ELP is the sphingosine-1-phosphate lyase (encoded by the gene lpp2128), an enzyme that in eukaryotes catalyzes 
Table 4 Genes encoding proteins containing eukaryotic motifs not previously described

\begin{tabular}{|c|c|c|c|c|c|c|}
\hline L. pneumophila & L. longbeachae & L. micdadei & L. hackeliae & L. fallonii (LLAP10) & Product & Eukaryotic motif \\
\hline Ipg0095/lpp0109 & 1103322 & $\operatorname{lmi1217}$ & Iha1000 & Ifa0120 & $\begin{array}{l}\text { Cytosolic IMP-GMP specific } \\
\text { 5'-nucleotidase }\end{array}$ & $\begin{array}{l}\text { HAD-superfamily hydrolase, } \\
\text { 5'-nucleotidase }\end{array}$ \\
\hline Ipg0301/lpp0379 & & & & Ifa0312\# & Protein of unknown function & DM9 repeat \\
\hline Ipg0971/lpp1033 & & & & & Apyrase & Nucleoside phosphatase \\
\hline $\operatorname{lpg} 1328^{\mathrm{a}} / \operatorname{lpp} 1283$ & & & & Ifa0766 & Protein of unknown function & Thaumatin \\
\hline Ipg1798//lpp1761 & 1100991 & & & & Protein of unknown function & RhoGTPase \\
\hline \multirow[t]{16}{*}{ Ipg1905/lpp1880 } & $1 / 01247$ & Imi2007 & Iha1318 & Ifa0744 & Apyrase & Nucleoside phosphatase \\
\hline & & & Iha0318 & & Protein of unknown function & DM9 repeat \\
\hline & 1103014 & & & Ifa2606 & Protein of unknown function & DM9 repeat \\
\hline & & & Iha0498 & & Protein of unknown function & Synaptobrevin/V-snare \\
\hline & & & & Ifa3165 & Protein of unknown function & Synaptobrevin/V-snare \\
\hline & 1100044 & & & & Protein of unknown function & Thaumatin \\
\hline & & Imil344 & & & Protein of unknown function & Thaumatin \\
\hline & & & Iha2013 & & Protein of unknown function & Thaumatin \\
\hline & & Imi0545 & Iha2362 & Ifa2256 & Protein of unknown function & DH/RhoGEF \\
\hline & 1100042 & & & Ifa0022 & Putative serine carboxypeptidase & Peptidase S28 \\
\hline & & & & Ifa1003 & Protein of unknown function & $\begin{array}{l}\text { Mitochondrial substrate/ } \\
\text { solute carrier repeat }\end{array}$ \\
\hline & & Imi0716 & & & $\begin{array}{l}\text { Protein of unknown function } \\
\text { [ARM repeat] }\end{array}$ & $\begin{array}{l}\text { Clathrin/coatomer_ } \\
\text { adaptine_like }\end{array}$ \\
\hline & & Imil174 & & & $\begin{array}{l}\text { Protein of unknown function } \\
\text { [HEAT-REPEAT DOMAIN] } \\
\text { [F-box domain] }\end{array}$ & $\begin{array}{l}\text { Clathrin/coatomer_ } \\
\text { adaptine_like }\end{array}$ \\
\hline & & Imi0037 & & & $\begin{array}{l}\text { Protein of unknown function } \\
\text { [coiled-coil] }\end{array}$ & RhoGTPase \\
\hline & & Imi0496 & & $1 f a 2567^{b}$ & Protein of unknown function & T-complex protein 10 \\
\hline & & Imi3046 & Iha3117 & & Protein of unknown function & Peptidase C65 \\
\hline
\end{tabular}

${ }^{\mathrm{a}} \mathrm{Gene}$ encoding a protein that has been demonstrated to be secreted by the Dot/lcm secretion system. ${ }^{\mathrm{b}}$ Orthologous proteins in which the corresponding motif is not present.

the irreversible cleavage of sphingosine-1-phosphate, and that has most likely been transferred horizontally from eukaryotes $[47,64,65]$. With the aim to detect proteins with higher similarity to eukaryotic proteins than to prokaryotic ones and for which we can suggest a eukaryotic origin through phylogenetic analysis, we have developed a pipeline that automatically extracts those proteins from the Legionella pan-genome with high similarity to eukaryotic proteins (for details see Materials and methods). Using this pipeline we identified 465 proteins as putative ELPs. For each of these proteins we constructed a phylogenetic tree that was curated and analyzed manually. However, for many of the ELPs a phylogenetic reconstruction did not allow clear demonstration of eukaryotic origin. Some aligned too poorly with their eukaryotic homologues or on just a small domain. This might be due to the fact that genomes of ciliated protozoa and amoeba, the known hosts of Legionella from which these ELPs are most likely acquired, are underrepresented in current databases. However, for 40 of the 465 proteins that are suggested to be of eukaryotic origin, the phylogenetic reconstruction clearly showed that they had been acquired by Legionella through horizontal gene transfer from eukaryotes (Table 5; Figure S9A-C in Additional file 14).

Among these proteins 27 had not been described before and 15 were identified in the newly sequenced species. A clear case of horizontal gene transfer from eukaryotes is GamA (Lpp0489), a glucoamylase that allows Legionella to degrade glycogen during intracellular replication in A. castellanii [66]. In addition to already characterized proteins, we identified promising candidates for host-pathogen interactions in this study - for example, a L. longbeachae protein containing a tubulin-tyrosine ligase domain (Llo2200; Figure S9A in Additional file 14), a motif involved in addition of a carboxy-terminal tyrosine to $\alpha$-tubulin as part of a tyrosination-detyrosination cycle that is present in most eukaryotic cells. This tyrosination process regulates the recruitment of microtubule-interacting proteins [67]. It is thus tempting to assume that Legionella is able to interfere with or to modulate the recruitment 
Table 5 Genes horizontally transferred from eukaryotic genomes to Legionella genomes

\begin{tabular}{|c|c|c|c|c|}
\hline L. pneumophila & L. longbeachae & L. micdadei & L. hackeliae & L. fallon \\
\hline Ipg0095/Ipp0109 & 1103322 & $\operatorname{Imi1217}$ & Iha1000 & Ifa0120 \\
\hline Ipg0422 /lpp0489 & 1102801 & & Iha1522 & Ifa2690 \\
\hline \multicolumn{5}{|l|}{ Ipg0971//pp1033 } \\
\hline Ipg1119//pp1120 & 1101016 & & & Ifa1837 \\
\hline \multicolumn{5}{|l|}{ Ipg1155//pp1157 } \\
\hline Ipg1565/lpp1522 & 1100920 & & Iha3027 & \\
\hline Ipg1675//pp1647 & $1 / 03277$ & Imi0628 & & Ifa1689 \\
\hline Ipg1905//pp1880 & 1101247 & Imi2007 & Iha1318 & Ifa0744 \\
\hline Ipg1950a/lpp1932 & 1101397 & & & \\
\hline Ipg2176//pp2128 & & & Iha1689 & Ifa3030 \\
\hline Ipg2556 /lpp2626 & 1102218 & & & \\
\hline lpg2911 & 1101568 & & & Ifa0029 \\
\hline $\operatorname{lpg} 2917$ & 1102243 & Imi0866 & Iha0637 & Ifa1734 \\
\hline \multirow[t]{11}{*}{ Ipg2951//pp3022 } & 1100076 & Imi3045 & Iha3116 & Ifa3723 \\
\hline & & Imi2545 & & Ifa1498 \\
\hline & 1100042 & & & Ifa0022 \\
\hline & 1101314 & & & \\
\hline & 1101320 & Imi2237 & Iha0011 & Ifa1070 \\
\hline & 1101999 & & & \\
\hline & 1102200 & Imi0200 & Iha3048 & Ifa0156 \\
\hline & 1102329 & & & \\
\hline & 1103082 & & & \\
\hline & 1103182 & & Iha1738 & \\
\hline & 1103288 & & & Ifa1897 \\
\hline
\end{tabular}

Imi0037

Imi0515

Imi0854

Imi1956

Imi1981

ralF

purC

cysk

cysk

cysteine synthase A, O-acetylserine sulfhydrolase A subunit

conserved protein of unknown function [FADPNR domain]

cysk

cysteine synthase A, O-acetylserine sulfhydrolase A subunit

protein of unknown function [SNARE domain]

protein of unknown function [ank domain] [Ras domain] [coiled-coil domain]

putative Dipeptidyl-peptidase ॥

conserved protein of unknown function [Leucine-rich repeat domain]

DWF 7-dehydrocholesterol reductase

putative Sphingomyelin phosphodiesterase

protein of unknown function [Tubulin-tyrosine ligase domain)

GTP-binding protein ypt1 [Ras small GTPase, Rab type]

protein of unknown function [Mitochondrial substrate carrier]

phosphoprotein phosphatase

protein of unknown function [Ras small GTPase, Rab type]

protein of unknown function [RhoGAP motif] [coiled-coil]

protein of unknown function [CAMP-binding domain-like]

protein of unknown function [Ras GTPase domain]

putative Adenosine deaminase

phosphoprotein phosphatase

protein of unknown function [Synaptobrevin]

protein of unknown function

membrane protein of unknown function

[Ras small GTPase, Rab type] 


\section{Table 5 Genes horizontally transferred from eukaryotic genomes to Legionella genomes (Continued)}

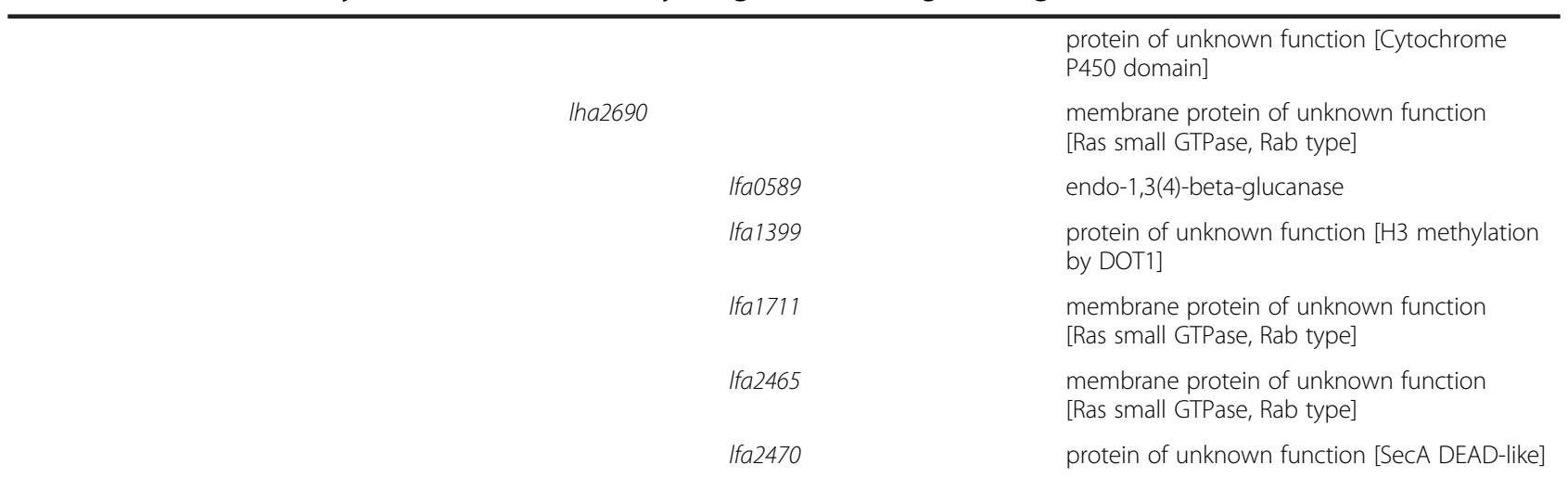

${ }^{\mathrm{a}}$ Gene encoding a protein that has been demonstrated to be secreted by the Dot/lcm secretion system.

of microtubule-interacting proteins in the host. Another example is the serine carboxypeptidase S28 family protein (Llo0042/Lfa0022; Figure 7). These proteins have been identified exclusively in eukaryotes and are active at low $\mathrm{pH}$, suggesting a function in the phagosome [68].

Taken together, each Legionella genome contains many different ELPs and proteins carrying eukaryotic domains that help Legionella to establish its intracellular niche. Some of these proteins are specific to one or other Legionella species but most are present in all of them, although these proteins are rarely real orthologues. This suggests that the acquisition of these proteins is important for Legionella to manipulate the host but that their horizontal acquisition has taken place on multiple occasions.

\section{Linking virulence properties and gene content}

When using THP-1 cells as a model for infection of human macrophages, not all Legionella species were able to infect and replicate (Figure 1A). These results correlated with the epidemiology of legionellosis where only certain Legionella species are isolated from human disease. With the aim of identifying the genetic bases conferring these differences, we searched for genes that were present in the strains that cause disease but absent in the ones that had not been isolated from humans. This comparative analysis showed that L. pneumophila, L. longbeachae and L. micdadei share 40 genes that are not present in any of the other species. Among those we identified the hyp operon (hypABFCDE - lpg2171-75), necessary for hydrogenase activity in $E$. coli and the cyanobacterium Synechocystis [69]. Legionella has additional downstream genes encoding for hydrogenases that are unique to these three species. This region is flanked by tRNA genes in L. micdadei and L. longbeachae, suggesting its acquisition by horizontal gene transfer.

Furthermore, a gene encoding a truncated hemoglobin (lpp2601) of group I called trHbN was identified as specific to the human pathogenic strains. Truncated hemoglobins are a family of small oxygen-binding heme proteins [70] that are ubiquitous in plants and present in many pathogenic bacteria such as Mycobacterium tuberculosis. Mycbacteria missing trHbNs are severely impaired for nitric oxide detoxification [71], and the expression of this gene is required for $M$. tuberculosis during macrophage infection [72]. The proteins of $M$. tuberculosis and L. pneumophila share 30\% identity and the important $\mathrm{TrHbN}$ residues are conserved in both, indicating a similar biochemical function. Furthermore, the $M$. tuberculosis trHbN shows $40 \%$ identity to its eukaryotic homologue in Tetrahymena thermophila and the Legionella protein $44 \%$ to the $T$. thermophila and $46 \%$ to the Paramecium tetraurelia protein. However, according to an in-depth phylogenetic analyses of truncated hemoglobins in prokaryotic and eukaryotic organisms, it seems that trHbNs are of prokaryotic origin and might have been transferred to eukaryotes [73]. Interestingly, the Lvh system is not part of the genes unique to L. pneumophila, L. longbeachae and L. micdadei as not all L. pneumophila strains contain it, but it is uniquely present only in these three species. Finally, of the more than 300 proteins described as translocated by the Dot/ Icm secretion system, only two, CegC4 (lpp2150/ lpg2200) and Lem25 (lpp2487/lpg2422), are exclusive to the three species found in human disease, but their function is not known yet.

Comparing L. pneumophila and L. longbeachae, the two species responsible for over $95 \%$ of human infections, to all other Legionella species, showed that 124 genes are specific to these human pathogenic Legionella. Among them are 38 substrates of the Dot/Icm secretion system, including RalF (lpp1932/lpg1950), SidJ (lpp2094/lpg2155), SidI (lpp2572/lpg2504), SdeC (lpp2092/lpg2153), SidE (lpp2572/lpg2504), SdcA (lpp2578/lpg2510) and CegC7 (lpp0286/lpg0227). In addition to the secreted substrates, iron availability seems to be important for the human pathogens as among the specific proteins several are related to iron scavenging or iron storage. These are homologues of PvcA and PvcB (lpp0236-lpp0237), the 


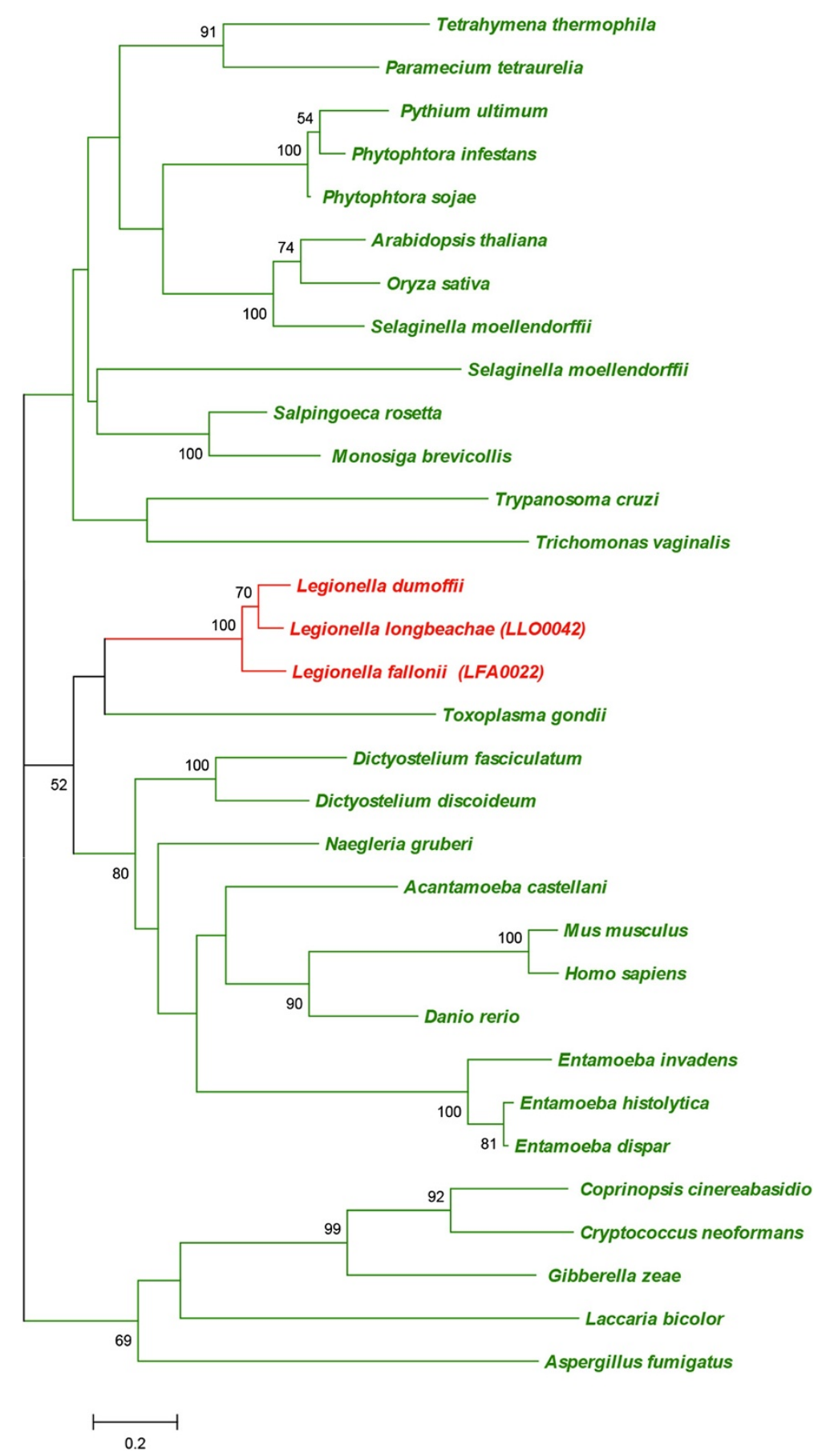

Figure 7 Phylogenetic analysis shows the eukaryotic origin of the carboxypeptidase S28 family protein (Llo0042/Lfa0022). The species belonging to bacteria and eukaryotes are shown in red and green, respectively. Numbers next to tree nodes correspond to bootstrap values. The bar at the bottom represents the estimated evolutionary distance.

siderophore pyoverdine that is involved in virulence and biofilm formation in the cystic fibrosis pathogen Pseudomonas aeuroginosa [74]. In Legionella these genes are highly expressed in sessile cells, suggesting their involvement in sessile growth [75]. Furthermore, a bacterioferritin (lpp2460) that is present also in L. micdadei but highly divergent is specific for the human pathogenic Legionella. Bacterioferritin plays a role in iron storage and is involved in protecting cellular components from oxidative damage, thereby playing a role in oxidative stress relief [76,77]. Furthermore, a gene coding for a homologue of the Yersinia pestis plasminogen activator (lpp2452) that 
was shown to create transient plasmin activity [78] and the phospholipase C (lpp1411) implicated in host killing in a G. mellonella model [79] are specific to L. pneumophila and L. longbeachae.

\section{Conclusions}

The first comprehensive analyses of five species of the genus Legionella and the comparison of the genomes of human disease-related strains with non-disease-related strains have provided new insights into the genomic specificities related to adaptation and host-pathogen interactions of this fascinating intracellular bacterium and have identified specific features of the major human pathogenic Legionella. Highly dynamic genomes that evolve through frequent horizontal gene transfer, mediated by many and diverse T4SSs and acquisition of different eukaryotic proteins and protein domains at multiple times and stages of their evolution that allow host subversion are a hallmark of this amoeba-associated bacterial genus. The major human-related Legionella species, L. pneumophila and L. longbeachae, contain a set of genes that seems to increase their successful infection of mammalian cells. The key to their success may be a better capacity to subvert host functions to establish a protective niche for intracellular replication due to a specific set of secreted effectors and a higher ability to acquire iron and to resist oxidative damage. The analysis of additional Legionella genomes and other intracellular pathogens may allow the future definition of the major common strategies used by intracellular pathogens to cause disease and to understand how environmental pathogens may evolve to become human pathogens.

\section{Materials and methods}

\section{Bacterial strains and sequence accession numbers}

The strains sequenced in this study were L. hackeliae strain ATCC35250 (EMBL accession number chromosome: PRJEB7321), L. micdadei ATCC 33218 (EMBL accession number chromosome: PRJEB7312) and L. fallonii strain LLAP-10 (ATCC700992; EMBL accession number chromosome: PRJEB7322) [25]. We obtained also the draft genome sequence of $L$. micdadei strain 02/42 (SRA accession number SRP047311), a clinical isolate from the Victorian Infectious Disease Research Laboratory (VIDRL). In addition, the genomes of Legionella species/strains that had been completely sequenced and published previously were included in the comparative analysis: L. pneumophila (strains Paris, Lens, Philadelphia, Corby, Lorraine and HL 0604 1035, Alcoy) $[7,8,12,14]$ and $L$. longbeachae strain NSW150 [9].

\section{Sequencing and assembly}

Strain L. micdadei 02/42 was sequenced using the Roche 454 GS-FLX platform, with Titanium chemistry and paired-end reads with an average insert size of $8.9 \mathrm{~kb}$. The resultant reads, with an average length of $215 \mathrm{bp}$, were assembled using Newbler 2.5.3 (Roche/454) into three scaffolds with a total genome size of 3,266,670 bp (largest scaffold 3,261,115 bp) and an average read coverage of 26. L. micdadeii ATCC33218, L. hackeliae and L. fallonii sequences were determined using a Sanger/ Illumina hybrid approach. For the Sanger approach sequencing reactions were performed using the ABI PRISM BigDye Terminator cycle sequencing ready reaction kit and a 3700 or a $3730 \mathrm{Xl}$ Genetic Analyzer (Applied Biosystems, Saint Aubin, Ille de France, France). For L. micdadei ATCC33218, L. hackeliae and L. fallonii, 33,042, 33,042 , and 36,240 sequences, respectively, from two libraries were determined. Assembly of the Sanger reads was done with the STADEN package in an iterative manner. We attempted to close remaining gaps with PCR products spanning repeats and regions recalcitrant to sequencing by testing several primer combinations for each gap. The final assemblies consisted of 36,084 reads and PCR products for L. micdadei ATCC33218, 33,085 for L. hackeliae, and 36,242 for L. fallonii. To finish the genome assembly each genome was in addition sequenced to a $60 \times$ coverage using an Illumina 2000 HiSeq sequencer and 36 bp reads. The Illumina reads and the programme Icorn [80] were used to correct the assembly and finish the genome.

\section{Annotation and genome comparison}

The newly sequenced genomes of $L$. fallonii, L. hackeliae and $L$. micdadei were integrated into the MicroScope platform [81] to perform automatic and expert annotation of the genes, and comparative analysis with the already sequenced and integrated L. pneumophila strains. MicrosScope annotation is based on a number of integrated bioinformatic tools: Blast on UniProt and specialized genomic data, InterPro, COG, PRIAM, synteny group computation using the complete bacterial genomes available at NCBI RefSeq, and so on (for more details see [82]). Orthologous groups were established using the program PanOCT [83] with the following parameters: e-value 1e-5, percent identity $\geq 30$, and length of match $\geq 65$. The programs Easyfig and BRIG $[84,85]$ were used for graphical representation of genome regions compared using BLAST. MAUVE [86] was used for aligning and comparing the $L$. micdadei genomes.

\section{A. castellanii and THP1 infection assays}

In brief, cultures of $A$. castellanii were grown in PYG712 medium ( $2 \%$ proteose peptone, $0.1 \%$ yeast extract, $0.1 \mathrm{M}$ glucose, $4 \mathrm{mM} \mathrm{MgSO}_{4}, 0.4 \mathrm{M} \mathrm{CaCl}_{2}, 0.1 \%$ sodium citrate dihydrate, $0.05 \mathrm{mM} \mathrm{Fe}\left(\mathrm{NH}_{4}\right)_{2}\left(\mathrm{SO}_{4}\right)_{2} \times 6 \mathrm{H}_{2} \mathrm{O}, 2.5 \mathrm{mM}$ $\mathrm{NaH}_{2} \mathrm{PO}_{3}, 2.5 \mathrm{mM} \mathrm{K} \mathrm{HPO}_{3}$ ) at $20^{\circ} \mathrm{C}$ for 3 days. Then amoeba were washed in infection buffer (PYG 712 
medium without proteose peptone, glucose, and yeast extract) and adjusted to $10^{5}$ to $10^{6}$ cells $/ \mathrm{ml}$. Stationary phase Legionella grown on BCYE (Buffer Charcoal Yeast Extract) agar and diluted in water were mixed with A. castellanii at a multiplicity of infection MOI of 0.1. After allowing invasion for $1 \mathrm{~h}$ at $20^{\circ} \mathrm{C}$ the $A$. castellanii layer was washed twice with infection buffer (start point of time-course experiment). Intracellular multiplication was monitored using a $300 \mu \mathrm{l}$ sample, which was centrifuged $(14,000 \mathrm{rpm})$ and vortexed to break up amoeba. The number of colony forming units (CFU) of Legionella was determined by plating on BCYE agar. The infections were carried out in duplicates.

The human monocytic cell line THP-1 was maintained in RPMI 1640 medium GlutaMAX medium (Gibco, Invitrogen, Saint Aubin, Ille de France, France), supplemented with $10 \%$ fetal bovine serum (BIOWEST, France Nuaille, Maine et Loire, France), in $5 \% \mathrm{CO}_{2}$ at $37^{\circ} \mathrm{C}$. For THP-1 infection, cells were seeded into 24-well tissue culture trays (Falcon, BD lab ware, Altrincham, Manchester, United Kingdom, England) at a density of $1.5 \times 10^{5}$ cells/well and pretreated with $10^{-8} \mathrm{M}$ phorbol 12-myristate 13-acetate (PMA) for $72 \mathrm{~h}$ in $5 \% \mathrm{CO}_{2}$ at $37^{\circ} \mathrm{C}$ to induce differentiation into macrophage-like adherent cells. Stationary phase Legionella were resuspended in RPMI 1640 serum free medium and added to THP-1 cell monolayers at an MOI of 10 . After $1 \mathrm{~h}$ of incubation cells were treated with $100 \mu \mathrm{g} \mathrm{ml}^{-1}$ gentamycin for $1 \mathrm{~h}$ to kill extracellular bacteria. Infected cells were then washed with phosphate-buffered saline (PBS) before incubation with serum-free medium. At 24, 48 and $72 \mathrm{~h}$ THP-1 cells were lysed with $0.1 \%$ TritonX-100. The amount of Legionella was monitored by counting the number of CFU determined by plating on BCYE agar. The infections were carried out in triplicate.

\section{Cyclase translocation assay}

The vector containing RalF-CyaA [29] was transformed into L. micdadei, L. hackeliae and L. fallonii and strain Paris wild type and its isogenic $\triangle d o t A .: K m$ mutant were used as positive and negative controls. Transformant strains were used to infect THP- 1 cells previously plated at $1 \times 10^{5}$ cells/well in 24-well tissue culture dishes and pre-treated with $10^{-8}$ M PMA. After $1 \mathrm{~h}$ and 30 minutes following infection cells were washed three times with cold PBS and lysed in $50 \mathrm{mM} \mathrm{HCl}, 0.1 \%$ Triton X-100. Lysates were boiled 5 minutes and neutralized with $0.5 \mathrm{M} \mathrm{NaOH}$. We then added 95\% cold ethanol and samples were spun for 5 minutes at maximum speed in a microcentrifuge. Supernatants were transferred in new $1.5 \mathrm{ml}$ tubes and vacuum dried, and cAMP concentrations were measured using the cAMP Biotrak Enzyme immunoassay System (Amersham, United Kingdom, England). Each value was calculated as means of two independent infections \pm standard deviations.

\section{Amoebae plate test}

Samples of suspended amoeba were applied to BCYE agar plates as described previously [19]. Stationary-phase bacterial cultures $(\mathrm{OD} 600>4.5)$ were adjusted to an identical OD600 (2.5), series of 10-fold dilutions in sterile $\mathrm{H}_{2} \mathrm{O}$ were prepared and $3 \mu \mathrm{l}$ of each dilution were spotted onto CYE plates both with amoeba and without amoeba (control plates) and incubated for 3 to 5 days at $30^{\circ} \mathrm{C}$ or $37^{\circ} \mathrm{C}$.

\section{Detection of new eukaryotic motifs in Legionella proteins}

To better define the term 'eukaryotic motifs' we searched for the already known EMs in all proteins present in the Pfam database and calculated their occurrence in eukaryotic proteins or prokaryotic proteins. The previously described EMs in Legionella showed an occurrence of about $99 \%$ in eukaryotic proteins and only $1 \%$ in prokaryotic ones, with the ankyrin repeats being the less restricted to eukaryotic proteins (85\%). The only exception is Sel-1 domains, which were considered as EMs. Sel-1 domains have now been shown to be highly present also in prokaryotes. However, since this domain is present in many substrates of the Dot/Icm system and it was shown to be implicated in host-pathogen interactions [87], it was taken into account. Based on the frequencies of the typical EMs present in Legionella we searched the Interpro database for all motifs that occur in eukaryotes at least to $85 \%$. Using this criterion we obtained 8,329 motifs that can be considered as eukaryotic. These motifs were searched in all proteins predicted in the different Legionella genomes. This approach identified 10 eukaryotic motifs previously not described in Legionella proteins.

\section{Detection of genes transferred from eukaryotes to Legionella}

To detect genes with putative eukaryotic origin we developed a pipeline based on several step filters. This pipeline was applied to one protein of each of the orthologous groups of the pan-proteome of the five studied species to avoid redundancy in the detection process with proteins of the same orthologous group. The first step consisted of discarding the protein families without significant similarity to eukaryotic sequences. This was achieved by a homology search using Blastp with an evalue cutoff of $\leq 10 \mathrm{e}^{-4}$ and a BLOSUM62 matrix with a representative protein of each group of orthologous families of the Legionella pan-genome against a database containing 83 genomes representative of all major eukaryotic phyla and certain viruses. In particular, members of Amoebozoa and other protist lineages that may be hosts for Legionella were included in this database. The results of the first filter led to the recovery of 2,669 proteins of the Legionella pan-genome with significant 
homology to eukaryotic sequences in the database. Then, among these 2,669 protein families those that have closer homologues in bacteria were discarded by searching for homologues against a database containing both eukaryotic and prokaryotic sequences using the same criteria. Only those that had at least a hit against a eukaryotic sequence among the first 25 hits were further selected. This step led to the selection of 465 protein families of the Legionella pan-genome representing ELP candidates. Finally, we carried out automatic phylogenetic reconstruction of these 465 proteins and their bacterial and eukaryotic homologues. The different steps of the pipeline were: (1) for each selected putative ELP the corresponding orthologues in other Legionella species analyzed where added if present; (2) each group of homologous sequences was aligned with MUSCLE [88]; (3) unambiguously aligned positions were automatically selected using the multiple alignment trimming program BMGE with low stringency parameters [89]; (4) preliminary maximum likelihood trees were obtained using FastTree [90]. We applied a strict filter to select only very likely ELPs. Then each of the 465 trees was manually inspected to select those where the Legionella sequences were branching within eukaryotes or were closer to eukaryotic sequences than to prokaryotic ones. This allowed identification of 40 Legionella proteins that aligned well with their eukaryotic homologues. For those having a sufficient number of eukaryotic homologues and a sufficient number of positions that could be selected after trimming, we proceeded to phylogenetic analysis by maximum likelihood using LG +4 gamma as the evolutionary model. Then, we selected those trees where the Legionella sequences were branching within eukaryotes or were closer to eukaryotic sequences than to prokaryotes. Finally, in order to verify the eventual existence of closer bacterial homologues or additional eukaryotic homologues from representatives not present in our local database, we performed a Blast on the non-redundant database at the NCBI. Alignments were obtained and trimmed, and trees reconstructed as described above.

\section{Phylogenetic reconstruction}

For phylogenetic reconstruction two different data sets were created: one based on the concatenated alignment of 29 housekeeping genes (lpp0086 (uvrB), lpp0152 (pgk), lpp0419 (rpoA), lpp0467 (ffh), lpp0575 (serS), lpp0749 (pros), lpp0791 (glyA), lpp1020 (lig), lpp1271 (cysS), lpp1399 (trpS), lpp1434 (aspD), lpp1534 (ruvB), lpp1738 (nrdA), lpp1765 (recA), lpp1830 (tig), lpp1837 (lepA), lpp2004 (metK), lpp2006 (dna)), lpp2013 (argS), lpp2020 (eno), lpp2662 (ftsZ), lpp2698 (uvrC), lpp2802 (dnaX), lpp2877 (recN), lpp2941 (metG), lpp3002 (rho), lpp3053 (atpD), lpp3055 (atpA), lpp3073 (thdF)) and another one based on all ortholgous genes among the studied species and C. burnetii as outgroup (816 genes). With these data sets the alignment of amino acids and the alignment of nucleotides based on the amino acid alignment were carried out. Individual genes/proteins were aligned with muscle and concatenated. The nucleotide alignments were cleaned using Gblocks [91]. Trees were constructed using both a distance method (neighbor-joining) implemented in the program MEGA [92] and a likelihood method using the software RaxML [93]. Bootstrap support was determined using 1,000 bootstrap replicates.

\section{Test for chitinase degradating activity}

According to Vadake [40], Whatman filter paper strips were cut to $5 \mathrm{~cm} \times 1 \mathrm{~cm}$. These strips were immersed and air-dried in a solution of p-nitroacetanilide $(5 \mathrm{~g}$ in $100 \mathrm{ml}$ of ethanol 100\%). The procedure was repeated three times to impregnate well the strips with pnitroacetanilide. L. fallonii and L. pneumophila (used as negative control) were grown in liquid medium for $24 \mathrm{~h}$ and $2 \mathrm{ml}$ of these cultures were transferred to a new sterile tube containing $2 \mathrm{ml}$ of fresh liquid media and the diagnostic strips. These cultures were grown for 2 days at $30^{\circ} \mathrm{C}$ for L. fallonii and $37^{\circ} \mathrm{C}$ for L. pneumophila. After 2 days the development of yellow color on the strip indicated the presence of deacetylase in the corresponding bacterial culture.

\section{Cellulose detection assays}

To visualize the production of cellulose, plates containing Legionella BCYE medium supplemented with calcofluor (5\%; fluorescent brightener 28; Sigma-Aldrich, Oakville, Ontario, Canada) were prepared. Drops of $5 \mu \mathrm{l}$ of liquid media containing L. fallonii grown for $72 \mathrm{~h}$ were spread on the plates and incubated at $30^{\circ} \mathrm{C}$ for $48 \mathrm{~h}$. The same procedure was carried out for L. pneumophila at $37^{\circ} \mathrm{C}$ as negative control. After incubation plates were visualized under a UV light source.

\section{Additional files}

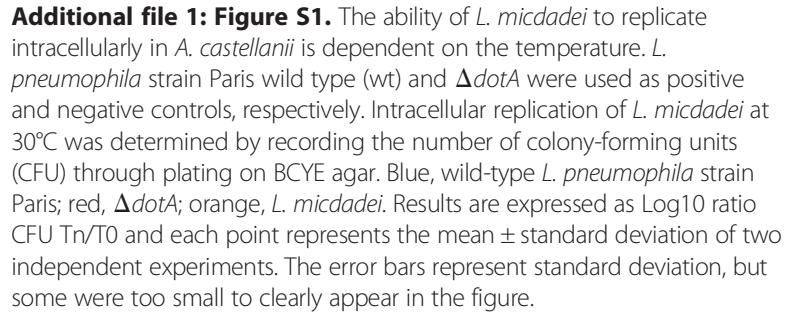

Additional file 1: Figure S1. The ability of L. micdadei to replicate intracellularly in $A$. castellanii is dependent on the temperature. $L$. pneumophila strain Paris wild type (wt) and $\Delta d o t A$ were used as positive and negative controls, respectively. Intracellular replication of $L$. micdadei at $30^{\circ} \mathrm{C}$ was determined by recording the number of colony-forming units (CFU) through plating on BCYE agar. Blue, wild-type L. pneumophila strain Paris; red, $\Delta$ dotA; orange, L. micdadei. Results are expressed as Log 10 ratio CFU Tn/TO and each point represents the mean \pm standard deviation of two independent experiments. The error bars represent standard deviation, but some were too small to clearly appear in the figure.

Additional file 2: Figure S2. The Dot/lcm-encoding genes are present in all Legionella species with variable homology. Graphical representation of Blastp comparisons of the genes encoding the Dot/lcm T4BSS in all strains/species used in this study. L. pneumophila strain Philadelphia 
genes were taken as query. Each color ring represents a species/strain and each segment in the ring a different gene. The intensity of the color indicates the percentage of amino acid identity. The percentages of identity and their correlation with the color intensity is given in the left panel. Gene names are given in the outside circle. Gene names in red indicate $100 \%$ amino acid identity.

Additional file 3: Figure S3. Genomic organization of the L. fallonii hopanoid encoding gene cluster. Blastx comparisons between clusters encoding genes for hopanoid biosynthesis in the species Streptomyces coelicolor and L. fallonii (LLAP10). The gray color code represents the Blast matches; the darker the gray the better the blast match. Protein names and their predicted functions are indicated below.

Additional file 4: Table S1. Putative mobile regions present in $L$. fallonii (LLAP10) containing a chloramphenicol acetyltransferase gene and a erythromycin esterase gene.

Additional file 5: Figure S4. L. fallonii shows chitinase degradation activity. Whatman paper strips dipped in a p-nitroacetanilide solution were introduced in liquid cultures of the species L. hackeliae, L. fallonii and L. pneumophila (used as negative control). After 2 days of growth the development of yellow color indicates the presence of chitin deacetylase activity in the bacterial culture.

Additional file 6: Table S2. Putative prophage region in the genome of L. micdadei strain ATCC33218.

Additional file 7: Figure S5. Genomic organization and comparison of the three conserved flagella gene clusters among the five Legionella species. Blastx comparison of these clusters in L. pneumophila, L. longbeachae, L. hackeliae, L. fallonii (LLAP10) and L. micdadei. (A) Flagellar region 1. (B) Flagellar region 2. (C) Flagellar region 3. The gray color code represents the Blast matches; the darker the gray the better the blast match.

Additional file 8: Figure S6. The L. pneumophila strain Paris plasmid is quasi-identical to the L. hackeliae plasmid. Chromosomal organization and Blastn comparison shows 100\% nucleotide identity except for the insertion of two transposase-encoding genes in strain Paris. The gray color code represents the Blast matches; the darker the gray the better the blast match.

Additional file 9: Figure S7. Comparison of F-type IV secretion systems present in the different Legionella species. Genomic organization and Blastx comparison between clusters encoding the F-type T4ASS, localized in the chromosome and on plasmids. Presence of the gene IVrC is indicated by a red dot. The gray color code represents the Blast matches; the darker the gray the better the blast match. Numbers indicate the chromosomal location.

Additional file 10: Figure S8. The Lvh-encoding region of the two sequenced L. micdadei strains is highly divergent. The Lvh T4ASS-encoding genes are highly conserved among all Legionella Blastx comparisons between the region encoding the Lvh system and the flanking genes in the two L. micdadei strains compared in this study. Blast matches are represented with gray lines that are darker as the blast match improves.

Additional file 11: Table S3. List of confirmed substrates of the Dot/ $\mathrm{Icm}$ secretion system.

Additional file 12: Table S4. List of eukaryotic organisms and viruses whose genomes have been selected for the construction of the eukaryotic genome database for Blast searches.

Additional file 13: Table S5 Orthologous genes encoding eukaryotic motifs in five Legionella species.

Additional file 14: Figure S9. Phylogenetic reconstruction for Legionella proteins that were probably transferred horizontally from eukaryotic organisms. Groups of homologous sequences were aligned with MUSCLE [89], unambiguously aligned positions were automatically selected using the multiple alignment trimming program BMGE [84] with low stringency parameters. After trimming, phylogenetic analysis was done using maximum likelihood.

\section{Abbreviations}

bp: base pair; BCYE: Buffer Charcoal Yeast Extract; CFU: colony forming units; ELP: eukaryotic-like protein; EM: eukaryotic motif; MOI: multiplicity of infection; PBS: phosphate-buffered saline; PMA: phorbol 12-myristate 13-acetate; SNP: single-nucleotide polymorphism; T4SS: type IV secretion system.

\section{Competing interests}

The authors declare that they have no competing interests.

\section{Authors' contributions}

LGV, CB, MS and KH designed the study. SJ, NKP and EH supplied material and expertise; GG and RJM performed genome sequencing; LGV and CR performed the genome annotation and analysis work; MR and JD undertook experiments; MN and SG performed phylogenetic analyses; CM set up the LegioScope database. LGV and CB drafted and wrote the manuscript. All authors contributed to and approved the final manuscript.

\section{Acknowledgements}

We would like to acknowledge P Glaser for critical reading of the manuscript and help from J Bernal with the calcoflour assay. The work in CB's laboratory received financial support from the Institut Pasteur, the Centre National de la Recherche (CNRS), the Fondation pour la Recherche Médicale (FRM) grant number DEQ20120323697, the French Region Ile de France (DIM Malinf) and from the ANR-10-PATH-004 project, in the frame of ERA-Net PathoGenoMics. The MicroScope platform received financial support from GIS IBiSA. MS acknowledges support from the Deutsche Forschungsgemeinschaft (DFG). ELH was supported by the Australian Research Council.

\section{Author details}

IInstitut Pasteur, Biologie des Bactéries Intracellulaires, 28, rue du Dr. Roux 75724 Paris, Cedex 15, France. ${ }^{2}$ CNRS UMR 3525, 75724 Paris, France. ${ }^{3}$ The ithree institute, University of Technology Sydney, Sydney, NSW 2007,

Australia. ${ }^{4} \mathrm{CIRI}$, International Center for Infectiology Research, Inserm, U1111, CNRS, UMR5308, Université Lyon 1, École Normale Supérieure de Lyon, Lyon F-69008, France. ${ }^{5}$ National Reference Center of Legionella, Hospices Civils de Lyon, 69677 Paris, France. ${ }^{6}$ Institut für Mikrobiologie, Technische Universität Braunschweig, Braunschweig, 38106, Germany. ${ }^{7}$ Cellular Interactions of Bacterial Pathogens, Centre for Biological Threats and Pathogens (ZBS2), Robert Koch-Institute, Nordufer 20, 13353 Berlin, Germany. ${ }^{8}$ Institut Pasteur, Unité Biologie Moléculaire du Gene chez les Extrêmophiles, Paris, 75015 , France. ${ }^{9} \mathrm{CEA} / \mathrm{DSV} / \mathrm{IG} /$ Genoscope \& CNRS UMR8030, Laboratoire d'Analyses Bioinformatiques en Génomique et Métabolisme, Evry 91000, France.

${ }^{10}$ Australian Animal Health Laboratories, CSIRO, Geelong, VIC 3220, Australia. ${ }^{11}$ Genomics, Institute for Biochemistry I, Medical Faculty, University of Cologne, D50931 Germany and Leibniz-Institute of Freshwater Ecology and Inland Fisheries, Berlin 10997, Germany. ${ }^{12}$ Department of Microbiology and Immunology, University of Melbourne at the Peter Doherty Institute for Infection and Immunity, Melbourne, VIC 3010, Australia.

Received: 25 July 2014 Accepted: 21 October 2014

Published online: 03 November 2014

\section{References}

1. Newton HJ, Ang DK, van Driel IR, Hartland EL: Molecular pathogenesis of infections caused by Legionella pneumophila. Clin Microbiol Rev 2010, 23:274-298.

2. Yu VL, Plouffe JF, Pastoris MC, Stout JE, Schousboe M, Widmer A, Summersgill J, File T, Heath CM, Paterson DL, Chereshsky A: Distribution of Legionella species and serogroups isolated by culture in patients with sporadic community-acquired legionellosis: an international collaborative survey. J Infect Dis 2002, 186:127-128.

3. Doleans A, Aurell H, Reyrolle M, Lina G, Freney J, Vandenesch F, Etienne J, Jarraud S: Clinical and environmental distributions of Legionella strains in France are different. J Clin Microbiol 2004, 42:458-460.

4. Svarrer CW, Uldum SA: The occurrence of Legionella species other than Legionella pneumophila in clinical and environmental samples in Denmark identified by mip gene sequencing and matrix-assisted laser desorption ionization time-of-flight mass spectrometry. Clin Microbiol Infect 2012, 18:1004-1009.

5. Alli OA, Zink S, von Lackum NK, Abu-Kwaik Y: Comparative assessment of virulence traits in Legionella spp. Microbiology 2003, 149:631-641.

6. Hägele S, Kohler R, Merkert H, Schleicher M, Hacker J, Steinert M: Dictyostelium discoideum: a new host model system for intracellular pathogens of the genus Legionella. Cell Microbiol 2000, 2:135-171. 
7. Cazalet C, Rusniok C, Bruggemann H, Zidane N, Magnier A, Ma L, Tichit M, Jarraud S, Bouchier C, Vandenesch F, Kunst F, Etienne J, Glaser P, Buchrieser $C$ : Evidence in the Legionella pneumophila genome for exploitation of host cell functions and high genome plasticity. Nat Genet 2004, 36:1165-1173

8. Chien M, Morozova I, Shi S, Sheng H, Chen J, Gomez SM, Asamani G, Hill K, Nuara J, Feder M, Rineer J, Greenberg JJ, Steshenko V, Park SH, Zhao B, Teplitskaya E, Edwards JR, Pampou S, Georghiou A, Chou IC, lannuccilli W, Ulz ME, Kim DH, Geringer-Sameth A, Goldsberry C, Morozov P, Fischer SG, Segal G, Qu X, Rzhetsky A, et al: The genomic sequence of the accidental pathogen Legionella pneumophila. Science 2004, 305:1966-1968.

9. Cazalet C, Gomez-Valero L, Rusniok C, Lomma M, Dervins-Ravault D, Newton HJ, Sansom FM, Jarraud S, Zidane N, Ma L, Bouchier C, Etienne J, Hartland $\mathrm{EL}$, Buchrieser C: Analysis of the Legionella longbeachae genome and transcriptome uncovers unique strategies to cause Legionnaires' disease. PLoS Genet 2010, 6:e1000851.

10. Kozak NA, Buss M, Lucas CE, Frace M, Govil D, Travis T, Olsen-Rasmussen M, Benson RF, Fields BS: Virulence factors encoded by Legionella longbeachae identified on the basis of the genome sequence analysis of clinical isolate D-4968. J Bacteriol 2010, 192:1030-1044.

11. D'Auria G, Jimenez-Hernandez N, Peris-Bondia F, Moya A, Latorre A: Legionella pneumophila pangenome reveals strain-specific virulence factors. BMC Genomics 2010, 11:181

12. Gomez-Valero L, Rusniok C, Jarraud S, Vacherie B, Rouy Z, Barbe V, Medigue C, Etienne J, Buchrieser C: Extensive recombination events and horizontal gene transfer shaped the Legionella pneumophila genomes. BMC Genomics 2011, 12:536.

13. Schroeder GN, Petty NK, Mousnier A, Harding CR, Vogrin AJ, Wee B, Fry NK, Harrison TG, Newton HJ, Thomson NR, Beatson SA, Dougan G, Hartland EL, Frankel G: Legionella pneumophila strain $130 \mathrm{~b}$ possesses a unique combination of type IV secretion systems and novel Dot/lcm secretion system effector proteins. J Bacterio/ 2010, 192:6001-6016.

14. Steinert M, Heuner K, Buchrieser C, Albert-Weissenberger C, Glockner G: Legionella pathogenicity: genome structure, regulatory networks and the host cell response. Int J Med Microbiol 2007, 297:577-587.

15. Brzuszkiewicz E, Schulz T, Rydzewski K, Daniel R, Gillmaier N, Dittmann C, Holland G, Schunder E, Lautner M, Eisenreich W, Lück C, Heuner K: Legionella oakridgensis ATCC 33761 genome sequence and phenotypic characterization reveals its replication capacity in amoebae. Int J Med Microbiol 2013, 303:514-528.

16. Wilkinson HW, Thacker WL, Steigerwalt AG, Brenner DJ, Ampel NM, Wing EJ: Second serogroup of Legionella hackeliae isolated from a patient with pneumonia. J Clin Microbiol 1985, 22:488-489.

17. Birtles RJ, Rowbotham TJ, Raoult D, Harrison TG: Phylogenetic diversity of intra-amoebal Legionellae as revealed by $16 \mathrm{~S}$ rRNA gene sequence comparison. Microbiology 1996, 142:3525-3530.

18. Neumeister B, Reiff G, Faigle M, Dietz K, Northoff H, Lang F: Influence of Acanthamoeba castellanii on intracellular growth of different Legionella species in human monocytes. Appl Environ Microbiol 2000, 66:914-919.

19. Albers U, Reus K, Shuman HA, Hilbi H: The amoebae plate test implicates a paralogue of IpxB in the interaction of Legionella pneumophila with Acanthamoeba castellanii. Microbiology 2005, 151:167-182.

20. MicroScope Microbial Genome Annotation \& AnalysisPlatform containing the sequenced and annotated Legionella genomes [https://www.genoscope.cns.fr/agc/microscope/about/collabprojects. php?P_id=27]

21. LegioList Integrated Genome database, containing the annotated genome sequences [http://genolist.pasteur.fr/LegioList/]

22. Coscolla M, Comas I, Gonzalez-Candelas F: Quantifying nonvertical inheritance in the evolution of Legionella pneumophila. Mol Biol Evol 2011, 28:985-1001.

23. Grattard F, Ginevra C, Riffard S, Ros A, Jarraud S, Etienne J, Pozzetto B: Analysis of the genetic diversity of Legionella by sequencing the 23S-5S ribosomal intergenic spacer region: from phylogeny to direct identification of isolates at the species level from clinical specimens. Microbes Infect 2006, 8:73-83.

24. Rubin CJ, Thollesson M, Kirsebom LA, Herrmann B: Phylogenetic relationships and species differentiation of 39 Legionella species by sequence determination of the RNase P RNA gene rnpB. Int J Syst Evol Microbiol 2005, 55:2039-2049.
25. Adeleke A, Pruckler J, Benson R, Rowbotham T, Halablab M, Fields B: Legionella-like amebal pathogens-phylogenetic status and possible role in respiratory disease. Emerg Infect Dis 1996, 2:225-230.

26. Adeleke AA, Fields BS, Benson RF, Daneshvar MI, Pruckler JM, Ratcliff RM, Harrison TG, Weyant RS, Birtles RJ, Raoult D, Halablab MA: Legionella drozanskii sp. nov., Legionella rowbothamii sp. nov. and Legionella fallonii sp. nov.: three unusual new Legionella species. Int I Syst Evol Microbiol 2001, 51:1151-1160.

27. Roy CR, Isberg RR: Topology of Legionella pneumophila DotA: an inner membrane protein required for replication in macrophages. Infect Immun 1997, 65:571-578

28. Berger $\mathrm{KH}$, Merriam JJ, Isberg RR: Altered intracellular targeting properties associated with mutations in the Legionella pneumophila dotA gene. Mol Microbiol 1994, 14:809-822.

29. Bardill JP, Miller JL, Vogel JP: IcmS-dependent translocation of SdeA into macrophages by the Legionella pneumophila type IV secretion system. Mol Microbiol 2005, 56:90-103.

30. Nagai $H$, Kagan JC, Zhu X, Kahn RA, Roy CR: A bacterial guanine nucleotide exchange factor activates ARF on Legionella phagosomes. Science 2002, 295:679-682.

31. Feldman M, Segal G: A specific genomic location within the $\mathrm{icm} / \mathrm{dot}$ pathogenesis region of different Legionella species encodes functionally similar but nonhomologous virulence proteins. Infect Immun 2004, 72:4503-4511

32. Feldman M, Zusman T, Hagag S, Segal G: Coevolution between nonhomologous but functionally similar proteins and their conserved partners in the Legionella pathogenesis system. Proc Natl Acad Sci U S A 2005, 102:12206-12211.

33. Luo ZQ, Isberg RR: Multiple substrates of the Legionella pneumophila Dot/ $\mathrm{Icm}$ system identified by interbacterial protein transfer. Proc Natl Acad Sci U S A 2004, 101:841-846.

34. Zusman T, Yerushalmi G, Segal G: Functional similarities between the $\mathrm{icm} /$ dot pathogenesis systems of Coxiella burnetii and Legionella pneumophila. Infect Immun 2003, 71:3714-3723.

35. Solano C, Garcia B, Valle J, Berasain C, Ghigo JM, Gamazo C, Lasa I: Genetic analysis of Salmonella enteritidis biofilm formation: critical role of cellulose. Mol Microbiol 2002, 43:793-808.

36. Zogaj X, Nimtz M, Rohde M, Bokranz W, Romling U: The multicellular morphotypes of Salmonella typhimurium and Escherichia coli produce cellulose as the second component of the extracellular matrix. Mol Microbiol 2001, 39:1452-1463.

37. Welander PV, Hunter RC, Zhang L, Sessions AL, Summons RE, Newman DK Hopanoids play a role in membrane integrity and $\mathrm{pH}$ homeostasis in Rhodopseudomonas palustris TIE-1. J Bacteriol 2009, 191:6145-6156.

38. Volkman JK: Sterols in microorganisms. Appl Microbiol Biotechnol 2003, 60:495-506.

39. Schmerk $C L$, Bernards MA, Valvano MA: Hopanoid production is required for low-pH tolerance, antimicrobial resistance, and motility in Burkholderia cenocepacia. J Bacteriol 2011, 193:6712-6723.

40. Vadake RS: Biotransformation of Chitin to Chitosan. United States Patent 5739015. 1998. United States Patent.

41. Campos-Gongora E, Ebert F, Willhoeft U, Said-Fernandez S, Tannich E: Characterization of chitin synthases from Entamoeba. Protist 2004, 155:323-330.

42. Grigor'ev AA, Bondarev VP, Borisevich IV, Darmov IV, Mironin AV, Zolotarev AG, Pogorel'skii IP, lanov DS: Temperate Legionella bacteriophage: discovery and characteristics. Zh Mikrobiol Epidemiol Immunobiol 2008, 4:86-88.

43. Lammertyn E, Vande Voorde J, Meyen E, Maes L, Mast J, Anné J: Evidence for the presence of Legionella bacteriophages in environmental water samples. Microb Ecol 2008, 56:191-197.

44. Molofsky AB, Byrne BG, Whitfield NN, Madigan CA, Fuse ET, Tateda K, Swanson MS: Cytosolic recognition of flagellin by mouse macrophages restricts Legionella pneumophila infection. J Exp Med 2006, 17:1093-1104

45. Ren T, Zamboni DS, Roy CR, Dietrich WF, Vance RE: Flagellin-deficient Legionella mutants evade caspase-1- and Naip5-mediated macrophage immunity. PLoS Pathog 2006, 2:e18.

46. Gomez Valero L, Runsiok C, Cazalet C, Buchrieser C: Comparative and functional genomics of Legionella identified eukaryotic like proteins as key players in host-pathogen interactions. Front Microbio/ 2011, 2:208. 
47. Gomez-Valero L, Buchrieser C: Genome dynamics in Legionella: the basis of versatility and adaptation to intracellular replication. Cold Spring Harb Perspect Med 2013, 3:a009993.

48. Lautner M, Schunder E, Herrmann V, Heuner K: Regulation, integrase-dependent excision, and horizontal transfer of genomic islands in Legionella pneumophila. J Bacteriol 2013, 195:1583-1597.

49. Bandyopadhyay P, Liu S, Gabbai CB, Venitelli Z, Steinman HM: Environmental mimics and the Lvh type IVA secretion system contribute to virulence-related phenotypes of Legionella pneumophila. Infect Immun 2007, 75:723-735.

50. Juhas M, Crook DW, Dimopoulou ID, Lunter G, Harding RM, Ferguson DJ, Hood DW: Novel type IV secretion system involved in propagation of genomic islands. J Bacteriol 2007, 189:761-771.

51. Wee BA, Woolfit M, Beatson SA, Petty NK: A distinct and divergent lineage of genomic island-associated type IV Secretion Systems in Legionella. PLoS One 2013, 8:e82221

52. Shohdy N, Efe JA, Emr SD, Shuman HA: Pathogen effector protein screening in yeast identifies Legionella factors that interfere with membrane trafficking. Proc Natl Acad Sci U S A 2005, 102:4866-4871.

53. Habyarimana F, Al-Khodor S, Kalia A, Graham JE, Price CT, Garcia MT, Kwaik YA Role for the Ankyrin eukaryotic-like genes of Legionella pneumophila in parasitism of protozoan hosts and human macrophages. Environ Microbiol 2008, 10:1460-1474.

54. Huang L, Boyd D, Amyot WM, Hempstead AD, Luo ZQ, O'Connor TJ, Chen C, Machner M, Montminy T, Isberg RR: The E Block motif is associated with Legionella pneumophila translocated substrates. Cell Microbio/ 2011, $13: 227-245$

55. Harding CR, Schroeder GN, Reynolds S, Kosta A, Collins JW, Mousnier A, Frankel G: Legionella pneumophila pathogenesis in the Galleria mellonella infection model. Infect Immun 2012, 80:2780-2790

56. Laguna RK, Creasey EA, Li Z, Valtz N, Isberg RR: A Legionella pneumophilatranslocated substrate that is required for growth within macrophages and protection from host cell deat. Proc Natl Acad Sci U S A 2006, 103:18745-18750.

57. Liu Y, Luo ZQ: The Legionella pneumophila effector SidJ is required for efficient recruitment of endoplasmic reticulum proteins to the bacterial phagosome. Infect Immun 2007, 75:592-603.

58. Eiserich JP, Estévez AG, Bamberg TV, Ye YZ, Chumley PH, Beckman JS, Freeman BA: Microtubule dysfunction by posttranslational nitrotyrosination of alpha-tubulin: a nitric oxide-dependent mechanism of cellular injury. Proc Natl Acad Sci U S A 1999, 96:6365-6370.

59. Acharya K, Pal AK, Gulati A, Kumar S, Singh AK, Ahuja PS: Overexpression of Camellia sinensis thaumatin-like protein, CSTLP in potato confers enhanced resistance to Macrophomina phaseolina and Phytophthora infestans infection. Mol Biotechnol 2013, 54:609-622.

60. Monteiro S, Barakat M, Picarra-Pereira MA, Teixeira AR, Ferreira RB: Osmotin and thaumatin from grape: a putative general defense mechanism against pathogenic fungi. Phytopathology 2003, 93:1505-1512.

61. Vodovar N, Vinals M, Liehl P, Basset A, Degrouard J, Spellman P, Boccard F, Lemaitre B: Drosophila host defense after oral infection by an entomopathogenic Pseudomonas species. Proc Natl Acad Sci U S A 2005, 102:11414-11419.

62. Sudhof TC, Baumert M, Perin MS, Jahn R: A synaptic vesicle membrane protein is conserved from mammals to Drosophila. Neuron 1989, 2:1475-1481.

63. McMahon HT, Mills IG: COP and clathrin-coated vesicle budding: different pathways, common approaches. Curr Opin Cell Biol 2004, 16:379-391

64. Degtyar E, Zusman T, Ehrlich M, Segal G: A Legionella effector acquired from protozoa is involved in sphingolipids metabolism and is targeted to the host cell mitochondria. Cell Microbiol 2009, 11:1219-1235.

65. Lurie-Weinberger MN, Gomez-Valero L, Merault N, Glockner G, Buchrieser C, Gophna U: The origins of eukaryotic-like proteins in Legionella pneumophila. Int J Med Microbiol 2010, 300:470-481.

66. Herrmann V, Eidner A, Rydzewski K, Bladel I, Jules M, Buchrieser C Eisenreich W, Heuner K: GamA is a eukaryotic-like glucoamylase responsible for glycogen- and starch-degrading activity of Legionella pneumophila. Int J Med Microbiol 2011, 301:133-139.

67. Szyk A, Piszczek G, Roll-Mecak A: Tubulin tyrosine ligase and stathmin compete for tubulin binding in vitro. J Mol Biol 2013, 425:2412-2414.

68. Tripathi LP, Sowdhamini R: Cross genome comparisons of serine proteases in Arabidopsis and rice. BMC Genomics 2006, 7:200.
69. Jacobi A, Rossmann R, Bock A: The hyp operon gene products are required for the maturation of catalytically active hydrogenase isoenzymes in Escherichia coli. Arch Microbiol 1992, 158:444-451.

70. Wittenberg JB, Bolognesi M, Wittenberg BA, Guertin M: Truncated hemoglobins: a new family of hemoglobins widely distributed in bacteria, unicellular eukaryotes, and plants. J Biol Chem 2002, 277:871-874.

71. Ouellet H, Ouellet Y, Richard C, Labarre M, Wittenberg B, Wittenberg J, Guertin M: Truncated hemoglobin HbN protects Mycobacterium bovis from nitric oxide. Proc Natl Acad Sci U S A 2002, 99:5902-5907.

72. Pawaria S, Lama A, Raje M, Dikshit KL: Responses of Mycobacterium tuberculosis hemoglobin promoters to in vitro and in vivo growth conditions. Appl Environ Microbiol 2008, 74:3512-3522.

73. Vuletich DA, Lecomte JT: A phylogenetic and structural analysis of truncated hemoglobins. J Mol Evol 2006, 62:196-210.

74. Wiens JR, Vasil Al, Schurr MJ, Vasil ML: Iron-regulated expression of alginate production, mucoid phenotype, and biofilm formation by Pseudomonas aeruginosa. MBio 2014, 5:e01010-e01013.

75. Hindre T, Bruggemann $H$, Buchrieser $C$, Hechard $Y$ : Transcriptional profiling of Legionella pneumophila biofilm cells and the influence of iron on biofilm formation. Microbiology 2008, 154:30-41.

76. Bou-Abdallah F, Lewin AC, Le Brun NE, Moore GR, Chasteen ND: Iron detoxification properties of Escherichia coli bacterioferritin. Attenuation of oxyradical chemistry. J Biol Chem 2002, 277:37064-37069.

77. Carrondo MA: Ferritins, iron uptake and storage from the bacterioferritin viewpoint. EMBO J 2003, 22:1959-1968.

78. Jarvinen HM, Laakkonen L, Haiko J, Johansson T, Juuti K, Suomalainen M, Buchrieser C, Kalkkinen N, Korhonen TK: Human single-chain urokinase is activated by the omptins PgtE of Salmonella enterica and Pla of Yersinia pestis despite mutations of active site residues. Mol Microbiol 2013, 89:507-517.

79. Aurass P, Schlegel M, Metwally O, Harding CR, Schroeder GN, Frankel G, Flieger A: The Legionella pneumophila Dot/lcm-secreted effector $\mathrm{PlcC} / \mathrm{CegC1}$ together with $\mathrm{PlcA}$ and $\mathrm{PlcB}$ promotes virulence and belongs to a novel zinc metallophospholipase $C$ family present in bacteria and fungi. J Biol Chem 2013, 288:11080-11092.

80. Otto TD, Sanders M, Berriman M, Newbold C: Iterative Correction of Reference Nucleotides (iCORN) using second generation sequencing technology. Bioinformatics 2010, 26:1704-1707.

81. Vallenet D, Belda E, Calteau A, Cruveiller S, Engelen S, Lajus A, Le Fevre F, Longin C, Mornico D, Roche D, Rouy Z, Salvignol G, Scarpelli C, Thil Smith AA, Weiman M, Médigue $C$ : MicroScope-an integrated microbial resource for the curation and comparative analysis of genomic and metabolic data. Nucleic Acids Res 2013, 41:D636-D647.

82. MicroScope Microbial Genome Annotation and Analysis Platform [https://www.genoscope.cns.fr/agc/microscope/home/index.php]

83. Fouts DE, Brinkac L, Beck E, Inman J, Sutton G: PanOCT: automated clustering of orthologs using conserved gene neighborhood for pan-genomic analysis of bacterial strains and closely related species. Nucleic Acids Res 2012, 40:e172.

84. Alikhan NF, Petty NK, Ben Zakour NL, Beatson SA: BLAST Ring Image Generator (BRIG): simple prokaryote genome comparisons. BMC Genomics 2011, 12:402

85. Sullivan MJ, Petty NK, Beatson SA: Easyfig: a genome comparison visualizer. Bioinformatics 2011, 27:1009-1010.

86. Darling AC, Mau B, Blattner FR, Perna NT: Mauve: multiple alignment of conserved genomic sequence with rearrangements. Genome Res 2004, 14:1394-1403.

87. Newton HJ, Sansom FM, Dao J, McAlister AD, Sloan J, Cianciotto NP, Hartland EL: Sel1 repeat protein LpnE is a Legionella pneumophila virulence determinant that influences vacuolar trafficking. Infect Immun 2007, 75:5575-5585.

88. Edgar RC: MUSCLE: a multiple sequence alignment method with reduced time and space complexity. BMC Bioinformatics 2004, 5:113.

89. Criscuolo A, Gribaldo S: BMGE (Block Mapping and Gathering with Entropy): a new software for selection of phylogenetic informative regions from multiple sequence alignments. BMC Evol Biol 2010, 10:210.

90. Price MN, Dehal PS, Arkin AP: FastTree 2-approximately maximum-likelihood trees for large alignments. PLoS One 2010, 5:e9490.

91. Castresana J: Selection of conserved blocks from multiple alignments for their use in phylogenetic analysis. Mol Biol Evol 2000, 17:540-552. 
92. Tamura K, Peterson D, Peterson N, Stecher G, Nei M, Kumar S: MEGA5: molecular evolutionary genetics analysis using maximum likelihood, evolutionary distance, and maximum parsimony methods. Mol Biol Evol 2011, 28:2731-2739

93. Stamatakis A, Aberer AJ, Goll C, Smith SA, Berger SA, Izquierdo-Carrasco F: RAxML-Light: a tool for computing terabyte phylogenies. Bioinformatics 2012, 28:2064-2066.

doi:10.1186/s13059-014-0505-0

Cite this article as: Gomez-Valero et al: Comparative analyses of Legionella species identifies genetic features of strains causing Legionnaires' disease. Genome Biology 2014 15:505.

\section{Submit your next manuscript to BioMed Central and take full advantage of:}

- Convenient online submission

- Thorough peer review

- No space constraints or color figure charges

- Immediate publication on acceptance

- Inclusion in PubMed, CAS, Scopus and Google Scholar

- Research which is freely available for redistribution 